\title{
SIRT5 is a proviral factor that interacts with SARS-CoV-2 Nsp14 protein
}

\author{
Authors \\ Marius Walter ${ }^{1 *}$, Irene P Chen ${ }^{2,3}$, Albert Vallejo-Gracia ${ }^{2,3}$, Ik-Jung Kim ${ }^{1}$, Olga Bielska ${ }^{1}$, Victor \\ L Lam $^{3}$, Jennifer M Hayashi ${ }^{2,3}$, Andrew Cruz ${ }^{1}$, Samah Shah ${ }^{1}$, John D Gross ${ }^{3,4}$, Nevan J \\ Krogan $^{2,3,4,5}$, Birgit Schilling ${ }^{1}$, Melanie Ott ${ }^{2,3}$, Eric Verdin ${ }^{1 \star}$. \\ ${ }^{1}$ Buck Institute for Research on Aging, Novato, CA, United States. \\ ${ }^{2}$ Gladstone Institutes, San Francisco, CA, United States. \\ ${ }^{3}$ University of California San Francisco, San Francisco, CA, United States. \\ ${ }^{4}$ Quantitative Biosciences Institute (QBI), University of California San Francisco, San Francisco, CA, United States. \\ ${ }^{5}$ QBI COVID-19 Research Group (QCRG), San Francisco, CA, United States.
}

*Correspondence to mwalter@buckinstitute.org, everdin@buckinstitute.org

\begin{abstract}
SARS-CoV-2 non-structural protein Nsp14 is a highly conserved enzyme necessary for viral replication. Nsp14 forms a stable complex with non-structural protein Nsp10 and exhibits exoribonuclease and N7-methyltransferase activities. Protein-interactome studies identified human sirtuin 5 (SIRT5) as a putative binding partner of Nsp14. SIRT5 is an NAD-dependent protein deacylase critical for cellular metabolism that removes succinyl and malonyl groups from lysine residues. Here we investigated the nature of this interaction and the role of SIRT5 during SARS-CoV-2 infection. We showed that SIRT5 stably interacts with Nsp14, but not with Nsp10, suggesting that SIRT5 and Nsp10 are parts of separate complexes. We found that SIRT5 catalytic domain is necessary for the interaction with Nsp14, but that Nsp14 does not appear to be directly deacylated by SIRT5. Furthermore, knock-out of SIRT5 or treatment with specific SIRT5 inhibitors reduced SARS-CoV-2 viral levels in cell-culture experiments. SIRT5 knock-out cells expressed higher basal levels of innate immunity markers and mounted a stronger antiviral response. Our results indicate that SIRT5 is a proviral factor necessary for efficient viral replication, which opens novel avenues for therapeutic interventions.
\end{abstract}




\section{Introduction}

Severe acute respiratory syndrome coronavirus 2 (SARS-CoV-2) is a pathogen of global concern that needs no further introduction. After cellular entry, SARS-CoV-2 hijacks the cellular machinery, and the viral proteins physically interact with hundreds of human proteins $(1-4)$. In most cases, however, the exact nature of the interactions and their functions and relevance during viral infection remain unknown.

SARS-CoV-2 encodes two large open reading frames, ORF1a and ORF1b, that are processed into 16 non-structural proteins after proteolytic cleavage by viral proteases. The 16 non-structural proteins, Nsp1 to Nsp16, are involved in every aspect of viral replication and are highly conserved in coronaviruses. Coronavirus Nsp14 protein is part of the replication-transcription complex and has two conserved domains with distinct functions. The N-terminal domain acts as a 3' to 5' exoribonuclease (ExoN), and the C-terminal domain displays RNA cap guanine N7-methyltransferase (MTase) activity (Fig. 1A) (5-8). The $\mathrm{N}$-terminal ExoN domain provides proofreading activity during RNA replication, allowing the removal of mismatched nucleotides introduced by the viral RNA polymerase (9-11). This proofreading activity ensures a high level of fidelity during RNA replication and is unique among RNA viruses $(12,13)$. Coronaviruses and related viruses in the order nidovirales have some of the largest genomes (26-32 kb) among known RNA viruses (14), and the acquisition of ExoN activity is thought to have allowed nidoviruses to evolve these large genomes $(9,15)$. The C-terminal MTase domain of Nsp14 is an S-adenosyl methionine (SAM)-dependent methyltransferase critical for viral RNA capping that methylates the $5^{\prime}$ guanine of the Gppp-RNA cap at the N7 position $(6,7)$. The 5 ' cap is important for viral mRNA stability and translation and for escaping host innate antiviral responses. Importantly, Nsp14 forms a stable complex with the non-structural protein Nsp10, a small zinc-binding co-factor with no reported enzymatic activity on its own $(7,10)$. Nsp10 binds and stabilizes the N-terminal ExoN domain of Nsp14 and is necessary for ExoN activity, but not for MTase activity. Interestingly, mutations that abolish ExoN activity cause a lethal phenotype in SARS-CoV-2 and MERS-CoV, but not in SARS-CoV or other coronaviruses (16), suggesting that ExoN has additional functions beyond its proofreading activity. Indeed, Nsp14 triggers translational shutdown, participates in evasion of innate immunity, activates proinflammatory signals, and mediates viral recombination (17-20).

Large-scale protein-protein interaction analyses of SARS-CoV-2 and human proteins revealed putative interacting partners for all of the SARS-CoV-2 proteins. Several independent studies, from us and from others, suggested that SARS-CoV-2 Nsp14 protein interacts with human sirtuin 5 (SIRT5) (1-4). Sirtuins are a family of conserved protein deacylases and mono-ADP-ribosyltransferases found in organisms ranging from bacteria to humans. Sirtuins use nicotinamide adenine dinucleotide (NAD) as a co-substrate and are important regulators of cellular metabolism and aging $(21,22)$. Most sirtuins act as NAD-dependent protein deacetylases, removing acetyl groups from lysine residues and, as such, tightly connect post-translational protein regulation with cellular metabolism. The seven 
bioRxiv preprint doi: https://doi.org/10.1101/2022.01.04.474979; this version posted January 5, 2022. The copyright holder for this preprint (which was not certified by peer review) is the author/funder, who has granted bioRxiv a license to display the preprint in perpetuity. It is made available under aCC-BY-NC-ND 4.0 International license.

Walter et al., 2022 - SIRT5/Nsp14

A

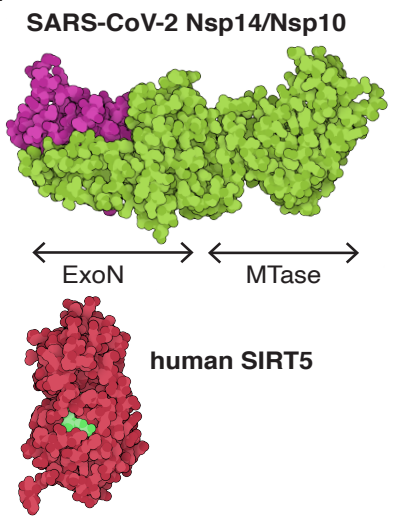

D

CETSA - SIRT5:

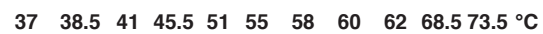

B

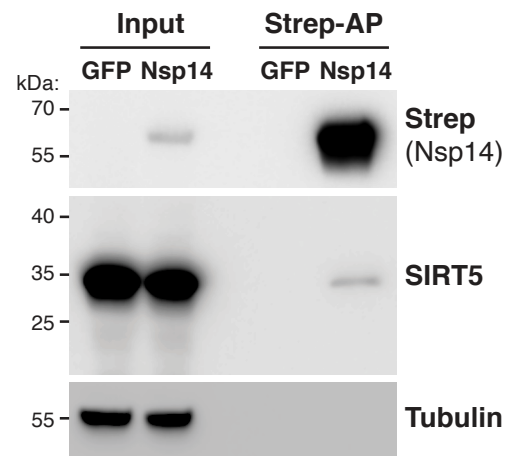

C
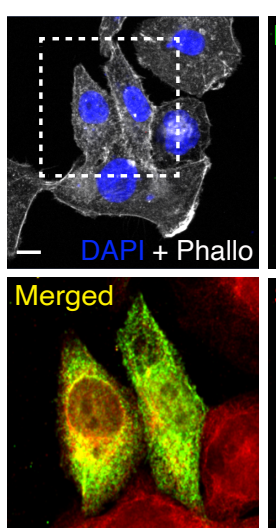

CETSA - Nsp14:

$3738.54145 .5515558606268 .573 .5^{\circ} \mathrm{C}$

Sirt5

Nsp14-Strep

+ Sirt5

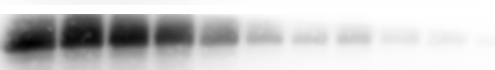

Nsp14-Strep

Nsp14-Strep

+ Sirt5

E

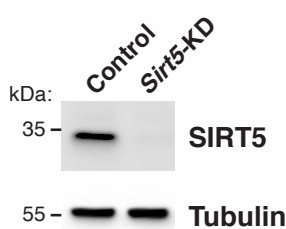

$\mathbf{F}$

SIRT
Nsp10-Fla
Nsp14-Stre
70

G

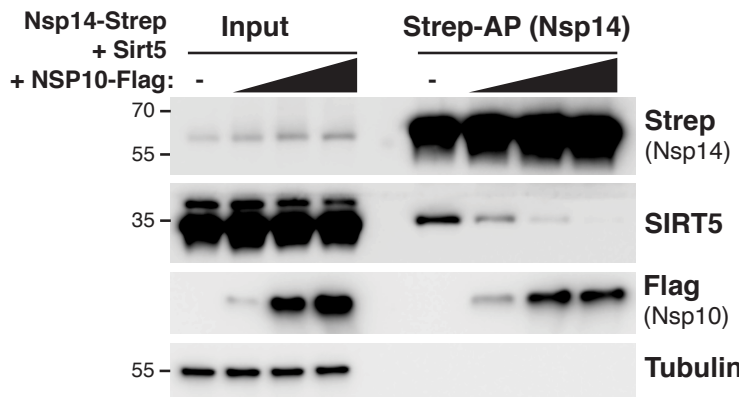

Figure 1: SARS-CoV-2 Nsp14 interact with human SIRT5.

A. Cartoon representation of the protein structure of Nsp14/Nsp10 (PDB 7N0B) and SIRT5 (PDB 3YIR) shows the Nsp14 N-terminal ExoN domain and C-terminal MTase domain. B. Affinity-purification of Nsp14-strep and co-purification of endogenous SIRT5 after transfection in HEK293T cells, as shown by western blot. C. Immunofluorescence of transfected Nsp14-Strep and endogenous SIRT5 in A549 cells. D. CETSA in HEK293T cells transfected with Nsp14-step and/or SIRT5, showing an increase in the stability of SIRT5 and Nsp14 by western blot. E. Western blot showing the absence of SIRT5 in Sirt5-KD HEK293T cells. F. Strep-tag affinity-purification or Flag-tag immunoprecipitation, followed by western blot, after transfection with Nsp14-strep, Nsp10-flag and SIRT5 expression constructs. SIRT5 does not interact with Nsp10. $0.5 \mu \mathrm{g}$ of each construct or of empty control plasmids were transfected in Sirt5-KD HEK293T cells in a six-well plate. G. Strep-tag affinity-purification and western blot after transfection of Nsp14-strep, SIRT5 and increasing concentrations of Nsp10-tag indicates competitive binding of SIRT5 and Nsp10. $0.5 \mu \mathrm{g}$ of Nsp14-strep and SIRT5 plasmid were used in a 6-well plate, with 0, 0.5, $1 \mathrm{or} 2 \mu \mathrm{g}$ of Nsp10-Flag. 
mammalian sirtuins (SIRT1-7) are found in different cellular compartments. They deacylate histones and transcriptional regulators in the nucleus and also specific proteins in the cytoplasm and in mitochondria. Sirtuins are crucial regulators of cellular metabolism and energy homeostasis and have emerged as key regulators of aging and age-related diseases.

SIRT5 is unique among the seven mammalian sirtuins. It is only a weak protein deacetylase, but it efficiently removes longer-chain acyl groups from proteins, such as succinyl, malonyl or glutaryl groups $(23,24)$. By preferentially catalyzing the removal of these negatively charged acidic modifications, SIRT 5 functions as the main cellular desuccinylase, demalonylase, and deglutarylase (24-26). SIRT5 is predominantly found in the mitochondria, but also exerts regulatory activity in the cytoplasm in several important metabolic processes, such as glycolysis, fatty acid oxidation and ketone body production $f(27)$. Despite elevated succinylation or malonylation levels in several tissues, no obvious phenotype or abnormalities are observed in Sirt5 knockout mice under basal conditions (28). The roles of SIRT5 in disease, infection, and aging, are unclear.

Here we investigated the role of SIRT5 during infection with SARS-CoV-2. We showed that SIRT5 stably interacts with Nsp14, but not with its cofactor Nsp10, and that SIRT5 catalytic activity is necessary for the interaction. Furthermore, knock-out or inhibition of SIRT5 reduced viral levels in cell-culture experiments, revealing that SIRT5 is a proviral factor necessary for efficient viral replication.

\section{Results}

\section{SARS-CoV-2 Nsp14 stably interact with human SIRT5}

Protein-protein interaction mass-spectrometry studies suggested that SARS-CoV-2 Nsp14 binds to SIRT5 (1-4). We first sought to confirm and characterize the nature of this interaction. We used a mammalian expression vector developed by Gordon et al. that contains Nsp14 with a 2xStrep affinity tag (Nsp14-strep) that can be used for affinity purification (1). Plasmids expressing Nsp14-strep or a GFP control were transfected into HEK-293T cells for 48 hours, and tagged proteins were purified by affinity purification using magnetic beads. Using western blots, we found that SIRT5 was specifically co-purifying with Nsp14, confirming published mass spectrometry results (Fig. 1B). Immunofluorescence in human alveolar basal epithelial A549 cells transfected with Nsp14 expression plasmid further showed that Nsp14 and SIRT5 co-localized into the same cellular compartments, with a predominantly cytoplasmic and peri-nuclear localization (Fig. 1C).

We next used Cellular Thermal Shift Assay (CETSA) to quantify the changes in the thermal stability of Nsp14 and SIRT5 in intact cells. The thermal stability of proteins changes upon ligand binding, and CETSA can be used to record the strength of the interaction in the physiological context. HEK-293T cells were transfected with plasmids expressing Nsp14 and SIRT5, either alone or in combination, and the shift in thermal stability was assessed by western blot (Fig. 1D). We observed an important increase in the stability of both proteins 
when they were co-transfected together. Nsp14, in particular, was poorly expressed and barely detectable when transfected alone, but a strong signal appeared in the presence of SIRT5. Overall, these initial observations showed that SIRT5 and Nsp14 were interacting in human cells and that SIRT5 strongly stabilized Nsp14 expression.

Nsp14 forms a stable complex with the small viral protein $\operatorname{Nsp10}(7,10)$. We thus tested whether SIRT5 also interacted with Nsp10, or whether they were parts of independent complexes. To eliminate endogenous expression of SIRT5, we generated a Sirt5 knockdown cell line (Sirt5-KD), using CRISPR-interference in HEK-293T cells (29). Several guide RNAs were tested, and one was selected for the rest of the study. SIRT5 was undetectable in this cell line (Fig. 1E). Expression plasmids for SIRT5, Nsp14-strep and Nsp10 with a Flag tag (Nsp10-Flag) were transfected alone or in combination for 48 hours in Sirt5-KD cells. Proteins were then co-purified either by strep affinity purification (Strep-AP) or Flag immunoprecipitation (Flag-IP) (Fig. 1F). Strep-AP confirmed that both SIRT5 and Nsp10 interacted with Nsp14. By contrast, pulling down Nsp10 by Flag-IP showed that only Nsp14 co-purified with Nsp10. This indicates that Nsp10 and SIRT5 do not interact. Besides, the SIRT5 signal after Strep-AP appeared to be lower in the presence of Nsp10, and we hypothesized that Nsp10 and SIRT 5 compete for Nsp14 binding. To test this hypothesis, Nsp14, SIRT5, and an increasing quantity of Nsp10 plasmids were co-transfected in Sirt5-KD cells. SIRT5 binding was lost with high concentrations of Nsp10 (Fig. 1G). Thus, SIRT5 and Nsp10 competitively bind Nsp14, and Nsp14/SIRT5 and Nsp14/Nsp10 likely form independent complexes.

\section{SIRT5 catalytic activity is necessary for the interaction with Nsp14}

SIRT5 is the main cellular desuccinylase, demalonylase, and deglutarylase, and a weak deacetylase (24-26). SIRT5 can physically bind to some of its enzymatic targets, such as Mitochondrial Antiviral Signaling Protein MAVS (30), mitochondrial serine hydroxymethyltransferase SHMT2 (31), or pyruvate kinase PKM2 (32). In these examples, SIRT5 both desuccinylates and binds the target protein as determined by co-immunoprecipitation. We thus hypothesized that SIRT5 could enzymatically modify Nsp14 and remove a putative succinyl, malonyl or glutaryl group.

To test this hypothesis, we determined if SIRT5 catalytic mutants bind Nsp14. Based on the structure of the SIRT5 catalytic domain and the homology with other sirtuins, we used or generated several expression constructs with mutations in conserved residues: $\mathrm{H} 158$ is catalytically required to abstract a proton from NAD, Q140 and I142 are involved in NAD binding, and Y102 and T105 interact with the extended acidic chains of succinyl or malonyl groups (Fig. 2A). H158, Q140 and I142 are universally conserved in sirtuins, but Y102 and T105 are specific to SIRT5 and mediate the specificity to longer-chain acidic groups $(23,33)$. Mutation of these residues is known (for H158Y, Y102F and R105M) or predicted (for Q140A and I142A, based on homology with other sirtuins) to abolish SIRT5 desuccinylation activity. After transfection of Nsp14-strep and SIRT5 mutants in Sirt5-KD cells, the binding of SIRT5 to Nsp14 was lost or severely reduced in most mutants (Fig. 2B). In particular, SIRT5 binding 
bioRxiv preprint doi: https://doi.org/10.1101/2022.01.04.474979; this version posted January 5, 2022. The copyright holder for this preprint (which was not certified by peer review) is the author/funder, who has granted bioRxiv a license to display the preprint in perpetuity. It is made available under aCC-BY-NC-ND 4.0 International license.

Walter et al., 2022 - SIRT5/Nsp14

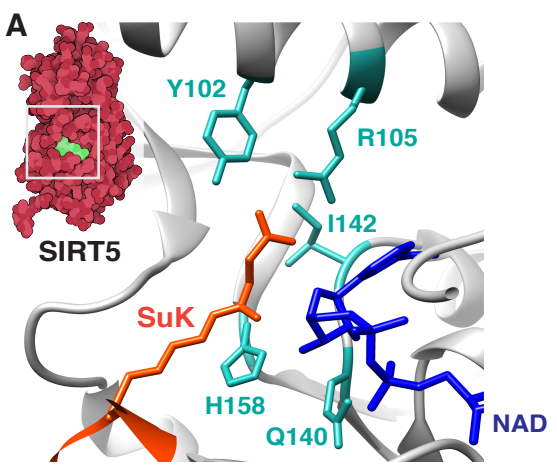

C

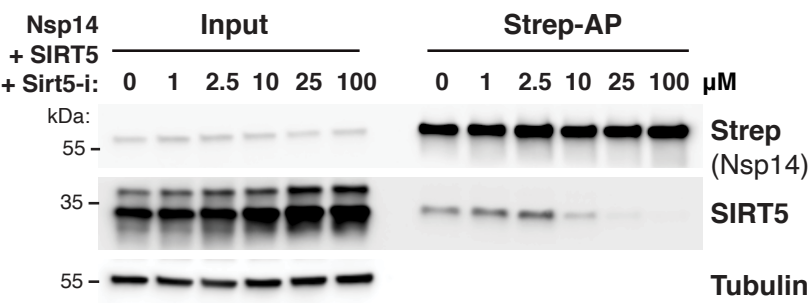

$\mathbf{B}$

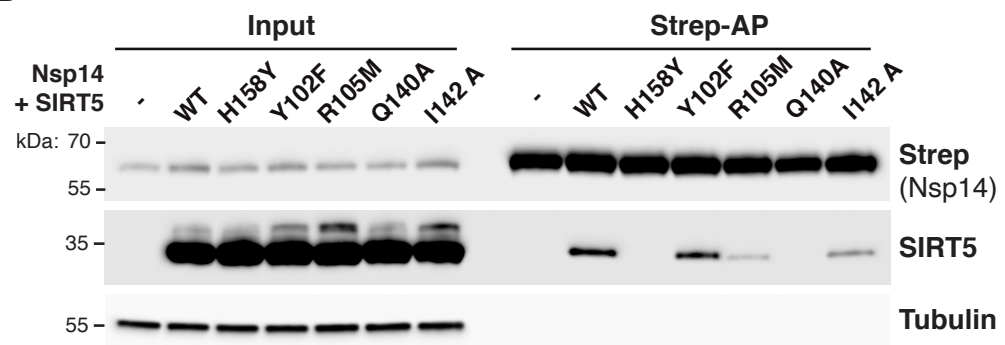

E
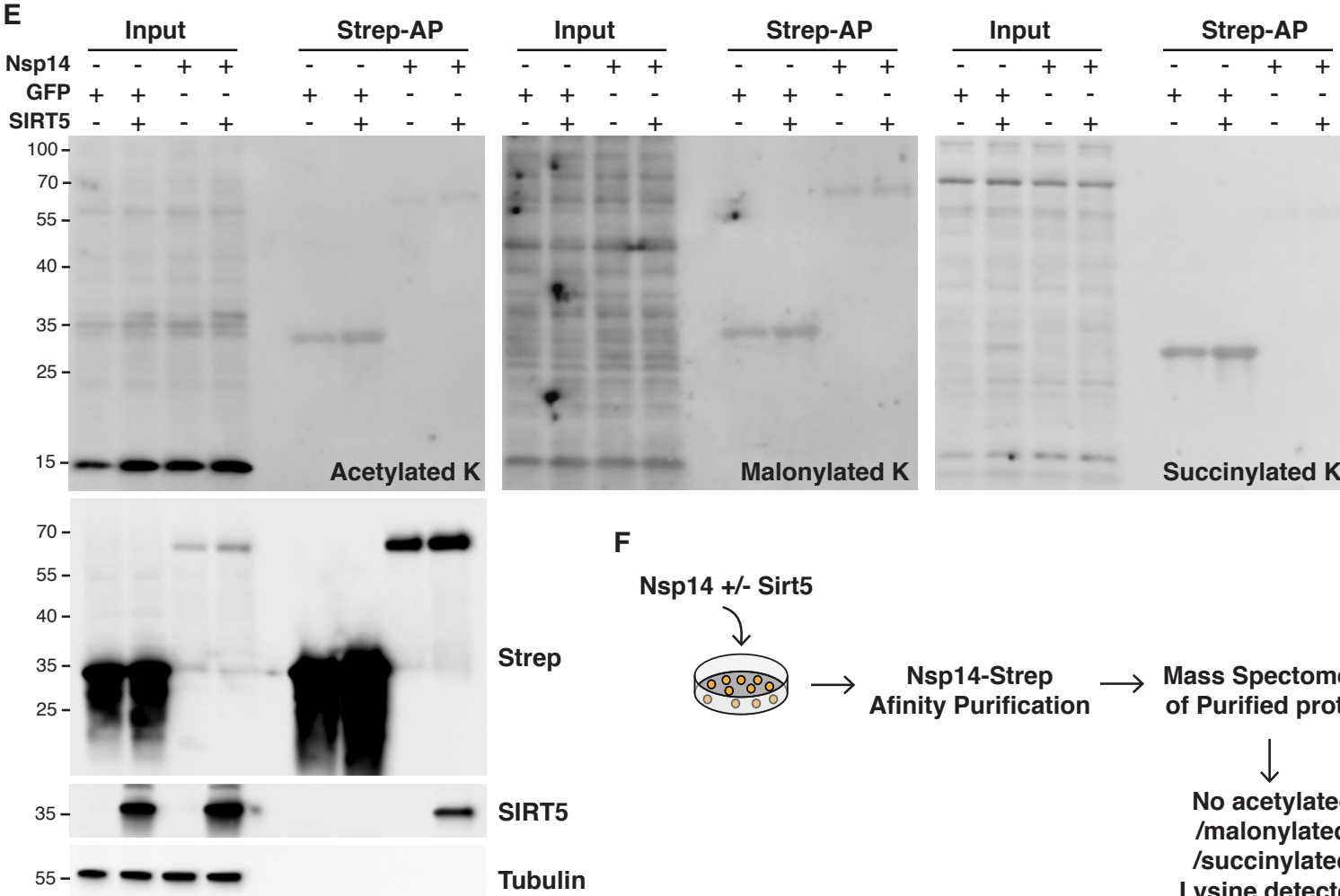

$\mathbf{F}$

Nsp14 +/- Sirt5

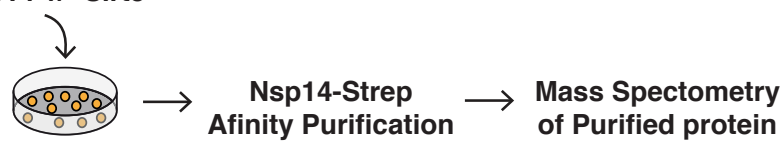

No acetylated

/malonylated

/succinylated

Lysine detected

Figure 2: SIRT5 catalytic activity is necessary to interact with Nsp14.

A. Cartoon representation of the protein structure of SIRT5 catalytic site in complex with cofactor NAD and succinylated lysine substrate (SuK), showing conserved residues mutated in panel B. B. Strep-tag affinity-purification and western blot after transfection of Sirt5-KD HEK293T cells with Nsp14-strep and SIRT5 catalytic mutants, showing that the interaction with Nsp14 is lost in several mutants. C. Strep-tag affinity-purification and western blot after transfection with Nsp14-strep and SIRT5, in Sirt5-KD HEK293T cells incubated with increasing concentrations of SIRT5 inhibitor Sirt5-i. High concentrations of Sirt5-i prevent the interaction. D. Strep-tag affinity-purification and western blot after transfection with Nsp14-strep and SIRT5, in Sirt5-KD HEK293T cells incubated with NAMPT FK866 inhibitor (low cellular NAD), FK866 and NMN, or NMN alone (high cellular NAD). SIRT5 binding strength correlated with NAD levels. E. Pan-acetylation, malonylation and succinylation in Sirt5-KD HEK293T total or Strep-purified proteins, after transfection with Nsp14-Strep, GFP-strep control and/or SIRT5. No specific lysine modifications could be detected. F. Summary of mass spectrometry experiments. Nsp14-strep proteins purified from Sirt5-KD HEK293T, with or without co-transfection with SIRT5, were analyzed by mass spectrometry. No acetylation, malonylation or succinylation modifications could be detected. 
completely disappeared in $\mathrm{H} 158 \mathrm{Y}$ and Q140A mutants, and only remained in significant amounts with the Y102F mutation. This result shows that an intact SIRT5 catalytic domain is necessary for the interaction with Nsp14.

To further establish that the catalytic activity of SIRT5 is necessary for the interaction with Nsp14, we used a recently described, potent and specific SIRT5 inhibitor (Sirt5-i) (34). This inhibitor has a published $\mathrm{IC}_{50}$ of $0.11 \mu \mathrm{M}$, and we measured an $\mathrm{IC}_{50}$ of $0.44 \mu \mathrm{M}$ in desuccinylation assays in vitro (Supplementary Fig. S1A). Sirt5-KD cells were transfected with Nsp14 and SIRT5 and incubated with increasing concentrations of Sirt5-i. The binding of SIRT 5 was lost at high concentrations of Sirt5-i, with the interaction almost absent at 25 and $100 \mu \mathrm{M}$ (Fig. 2C). This observation again suggested that the SIRT5 catalytic activity was necessary for the interaction. Notably, until now, Sirt5-i had not been characterized in cell culture. This experiment suggested that Sirt5-i could be efficiently used in cells, with a putative $\mathrm{IC}_{50}$ of approximately $10 \mu \mathrm{M}$. Next, we tested whether the interaction depended on cellular NAD levels. NAD is a necessary co-substrate, and SIRT5 activity is highly correlated with cellular NAD levels. In cells, most NAD is synthesized through the NAD salvage pathway (35). Treating cells with the NAMPT inhibitor FK866 blocks the rate-limiting step in the pathway and rapidly depletes NAD levels, and supplementing cells with the NAD precursor nicotinamide mononucleotide (NMN) rescues the depletion (36) (Supplementary Fig. S1B). Sirt5-KD cells were transfected for 24 hours with Nsp14 and SIRT5 and then for an additional 24 hours with FK866 and/or NMN (Fig. 2D). SIRT5 binding was highly reduced in the presence of FK866 (low NAD levels), but the binding was rescued in presence of NMN, and appeared slightly stronger when cells were treated with NMN alone (high NAD levels). This finding indicated that the interaction of Nsp14 and SIRT5 positively correlated with cellular NAD levels. Altogether, by directly inhibiting SIRT 5 or modulating the level of its co-substrate NAD, these experiments confirmed that SIRT5 catalytic activity was necessary for the interaction with Nsp14.

Finally, we determined if Nsp14 was directly modified by SIRT5 and if we could detect changes in the levels of acetylation, succinylation or malonylation. Sirt5-KD cells were transfected with SIRT5, Nsp14 and/or a GFP expression control. After affinity purification, pan-acetylation/succinylation/malonylation antibodies were used to detect changes in the levels of different lysine modifications (Fig. 2E). Independent of the presence of SIRT5, we detected no measurable changes in acetylation, succinylation or malonylation, either among input or purified proteins. Weak bands were observed for purified GFP and Nsp14, but the intensity of the signal was not affected by the presence or absence of SIRT5, suggesting that it is not specific. Pan-modification antibodies are often not very sensitive, and we tested whether we could detect changes in lysine modifications by mass spectrometry.

Nsp14-strep was transfected in Sirt5-KD cells, purified by affinity purification, and analyzed by mass spectrometry. The experiment was done with and without SIRT5 co-transfection, in three biological replicates each. In both conditions, Nsp14 had high coverage (65\% coverage in presence of SIRT5, 88\% without SIRT5), and we confirmed the interaction of Nsp14 with the two human proteins GLA and IMDH2 as reported (1) (Supplementary table S1). However, 
and despite the high coverage and high quality of the data, we found no acetylated, succinylated, malonylated or glutarylated sites. We detected a previously characterized phosphorylation site at serine 56 (37) and a nitrosylation site on tyrosine 351 . Since SIRT5 is the only known cellular desuccinylase, demalonylase, and deglutarylase, we had hoped that analyzing Nsp14 post-translational modifications in the presence or absence of SIRT5 would reveal changes in the level of these acylations marks. On the contrary, the absence of detectable acetylation, succinylation, malonylation and glutarylation suggested that Nsp14 was not directly modified by SIRT5. This series of experiments revealed that, even though SIRT5 catalytic activity is necessary for the interaction with Nsp14, Nsp14 did not appear to be a direct target of SIRT5.

\section{Nsp14 also interact with SIRT1}

The seven human sirtuins share conserved domains, and we tested whether Nsp14 also interacts with the other sirtuins. We found that Nsp14 interacted with SIRT1, but not with SIRT2, 3, 6 or 7 (Fig. 3A). We could not investigate the interaction with SIRT4 because we lacked a specific antibody. The signal from SIRT1 was specific but appeared weaker than with SIRT5, which might explain why it was not detected by mass spectrometry (1). Interestingly, and as we observed with SIRT5, mutating SIRT1 catalytic domain or treating cells with the SIRT1 inhibitor Ex-527 eliminated the interaction (Fig. 3B, C). This finding suggested again that SIRT1 catalytic activity is necessary for the interaction with Nsp14. By contrast, treating cells with the specific SIRT1 activator SRT1720 or the non-specific activator Resveratrol had no measurable effect on Nsp14 binding, and both activators were toxic to cells at high concentrations (Fig. 3D, E).

To uncover a putative molecular function of the Nsp14-SIRT5 interaction, Nsp14 and SIRT5 were expressed in $E$. coli and purified. Unfortunately, and even though the complex could be readily observed in mammalian cells, we could not reconstitute it in vitro with purified proteins, either by column chromatography or gel electrophoresis. This was the case when the two proteins were expressed separately and purified, or when co-expressed in $E$. coli. This observation suggests that the in vitro conditions that we used were inadequate and that additional parameters in mammalian cells were likely missing, such as other protein cofactors, post-translational modifications, or specific buffer conditions. Even though the complex could not be observed in vitro, we determined if the enzymatic activities of SIRT 5 or Nsp14 were perturbed when in presence of each other. We measured the desuccinylation activity of SIRT5 alone or when incubated with increasing concentrations of Nsp14, using an in vitro desuccinylation assay. We detected no changes in desuccinylation activity in presence of Nsp14, whereas adding known inhibitors, such as nicotinamide or the specific SIRT5 inhibitor Sirt5-i, completely inhibited the activity (Fig. 3F). Similarly, we used a methyltransferase assay to characterize the MTase activity of Nsp14 in presence of SIRT5. This assay measures the conversion of the methyl donor S-adenosyl methionine (SAM) into S-adenosyl homocysteine and can be used to measure the activity of any SAM-dependent methyltransferase. Nsp14 methylates the mRNA cap and Nsp14 was incubated with an unmethylated GpppG cap-analog, in presence of SAM, NAD and increasing concentrations of 
bioRxiv preprint doi: https://doi.org/10.1101/2022.01.04.474979; this version posted January $5,2022$. The copyright holder for this preprint (which was not certified by peer review) is the author/funder, who has granted bioRxiv a license to display the preprint in perpetuity. It is made available under aCC-BY-NC-ND 4.0 International license.

Walter et al., 2022 - SIRT5/Nsp14

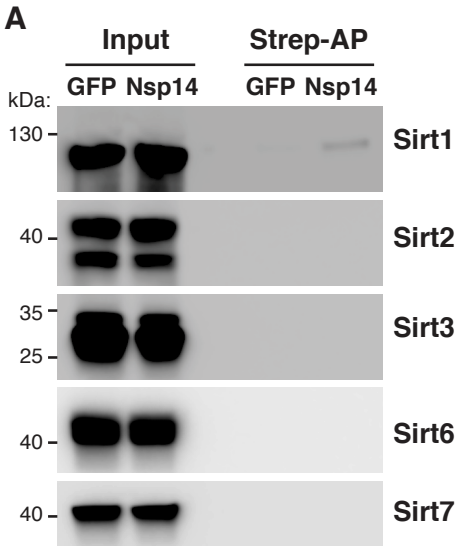

B

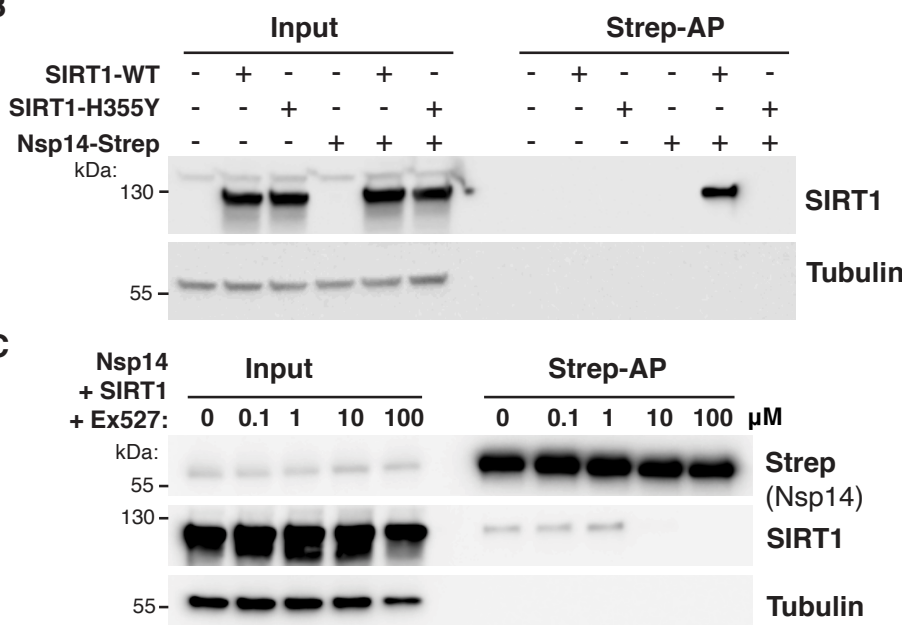

D

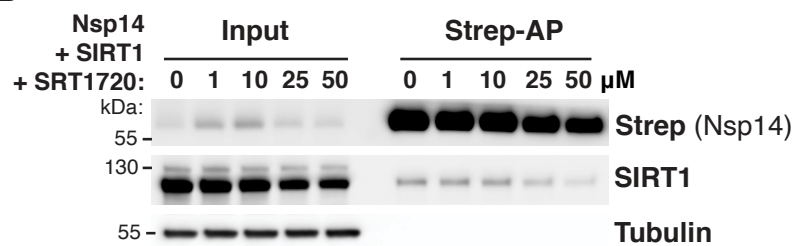

E

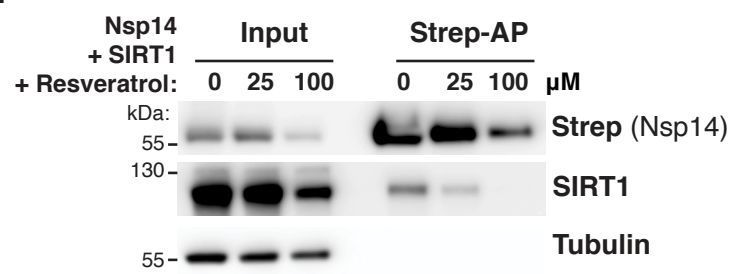

$\mathbf{F}$

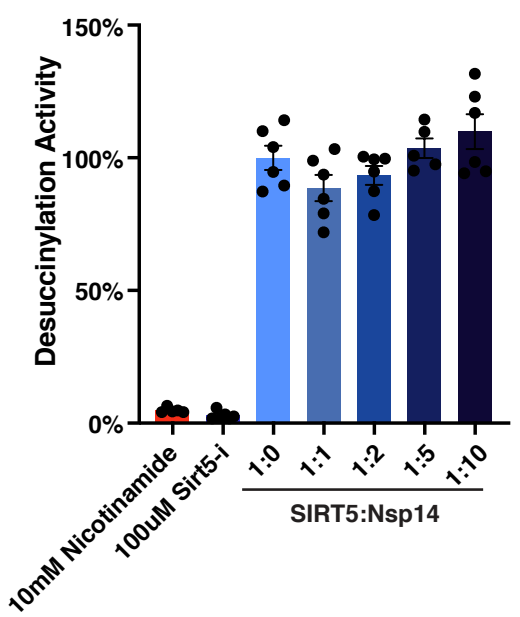

G

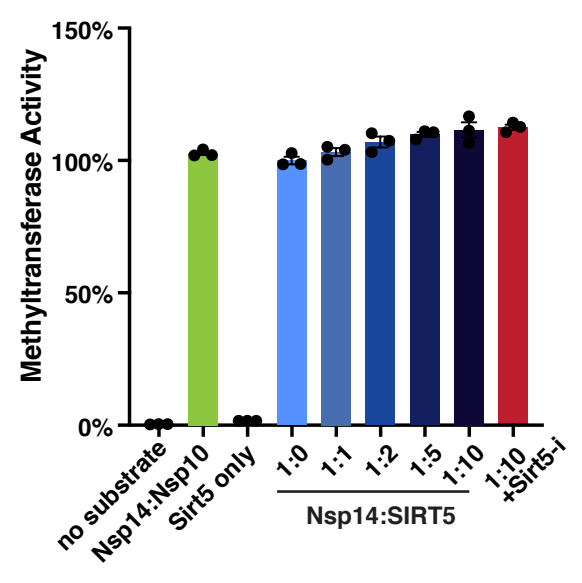

Figure 3: SARS-CoV-2 Nsp14 interact also with human SIRT1.

A. Co-purification of endogenous sirtuins SIRT1, 2 , 3, 6 and 7 after transfection of Nsp14-strep in HEK293T cells, as shown by western blot. Loading and purification controls are the same as in Fig. 1B. B. Strep-tag affinity-purification and western blot after transfection of HEK293T cells with Nsp14-strep and SIRT1 WT and H355Y catalytic mutant, showing that the interaction with Nsp14 is lost in H355Y mutant. C. Strep-tag affinity-purification and western blot after transfection with Nsp14-strep and SIRT1, in HEK293T cells incubated with increasing concentrations of SIRT1 inhibitor Ex-527. High concentrations of Ex-527 prevent the interaction. D-E. Strep-tag affinity-purification and western blot after transfection with Nsp14-strep and SIRT1, in HEK293T cells incubated with increasing concentrations of SIRT1 specific activator SRT1720 (D) or non-specific activator resveratrol (E). Both drugs were cytotoxic at high concentration and didn't affect SIRT5 binding. F. In vitro desuccinylation activity of purified SIRT5 incubated with increasing concentrations of Nsp14, showing no effect. G. In vitro methyltransferase activity of purified Nsp14 incubated with increasing concentrations of SIRT5, showing no specific effect. Unmethylated GpppG cap-analog was used as a substrate. 
SIRT5 (Fig. 3G). The Nsp14/Nsp10 complex and Nsp14 alone had similar activities, as predicted. We detected a small $10 \%$ increase of methyltransferase activity with excess SIRT5, but this increase persisted in presence of the SIRT5 inhibitor Sirt5-i. Since we showed above that Sirt5-i disrupted the interaction (Fig. 3G), this small increase might not be specific. Besides, Nsp14 ExoN activity depends on Nsp10, and Nsp14 has no ExoN activity by itself. We showed that Nsp10 and SIRT5 are parts of separate complexes (Fig. 1F-G), suggesting that the Nsp14/SIRT5 complex is very unlikely to have any ExoN activity either. Overall, these enzymatic assays failed to uncover a clear molecular function of the Nsp14/SIRT5 complex.

\section{SIRT5 is a proviral factor}

We next wanted to examine the role of SIRT5 during SARS-CoV-2 infection. We generated a Sirt5 knockout (Sirt5-KO) in A549 cells overexpressing ACE2 (A549-ACE2), using CRISPR-Cas9 editing and three commercially designed guide RNAs. The knockout was generated by transfecting cells with Cas9-gRNA ribonucleoproteins (RNPs), and cells transfected with Cas 9 alone and no guide RNAs were used as wild-type (WT) controls. As expected, SIRT5 was undetectable in Sirt5-KO cells (Fig. 4A). WT and Sirt5-KO cells were infected with SARS-CoV-2 (Wuhan strain, USA/WA-1/2020 isolate) at a multiplicity of infection (MOI) of 0.1 and 1 , and viral RNA was quantified by RT-qPCR after 3 days (Fig. 4B). At both MOIs, we observed a significant 2-3-fold decrease of SARS-CoV-2 mRNA in Sirt5-KO cells $(p<0.0001$ and $p=0.0002$ at $\mathrm{MOI}=0.1$ and 1 , respectively, by ANOVA). These results were confirmed by plaque assay, and we measured a significant fourfold decrease of viral titers in Sirt5-KO cells (Fig. 4C, $p=0.0049$, ANOVA). These observations suggested that SIRT5 is necessary for SARS-CoV-2 replication and/or propagation.

To confirm this result, A549-ACE2 cells were infected with SARS-CoV-2 in presence of the specific inhibitor Sirt5-i. Sirt5-i caused no measurable cytotoxicity (Fig. 4D) and significantly reduced cell-associated viral mRNA levels by almost twofold (Fig. 4E, $p=0.0001$ and $p<0.0001$ at 25 and $100 \mu \mathrm{M}$, respectively, ANOVA). Viral titers measured by plaque assay were also reduced in presence of Sirt5-i inhibitor, albeit not significantly (Fig. 4F). Human lung-cancer cells Calu-3 endogenously express ACE2 and are often considered a better model for SARS-CoV-2 infection. Calu-3 cells were infected with SARS-CoV-2 and treated with SIRT5 inhibitor Sirt5-i. Viral RNA levels were significantly reduced, with a twofold reduction in viral mRNA at $100 \mu \mathrm{M}$ (Fig. 4G, $\mathrm{p}=0.011$, ANOVA). Viral titers measured by plaque assay were also reduced in Calu3 cells in presence of Sirt5-i inhibitor (Fig. 4H). Notably, viral infections and plaque assays often have important inter- and intra-experiment variations, making it difficult to compare biological replicates that are not generated side-by-side. This explains the extremely high range of data points observed in Fig. 4F and $\mathrm{H}$, with the "batch" effect (for example the same experiment repeated on different days) accounting for up to $65 \%$ of the experimental variance (two-way ANOVA, $p<0.0001$ ). To correct for this batch effect, we calculated a plaque assay Z-score, using the pooled variance between experiments, and used this standardized measure to accurately analyze the effect of SIRT5 knockout and inhibition (Fig. 4I). This confirmed that viral titers were significantly 
Walter et al., 2022 - SIRT5/Nsp14

A

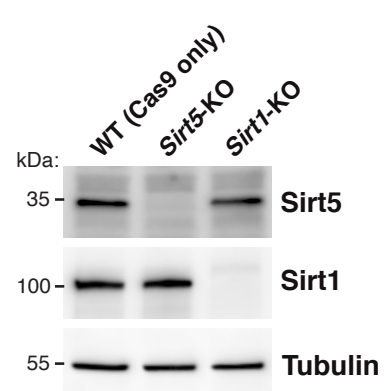

D

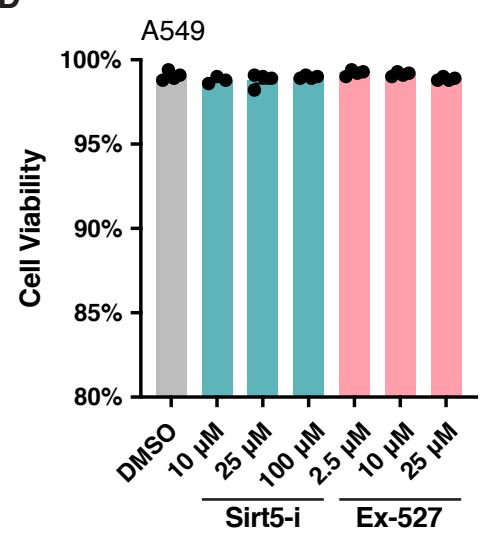

G

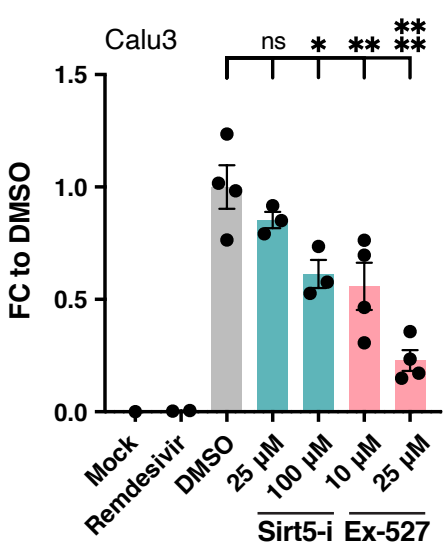

J

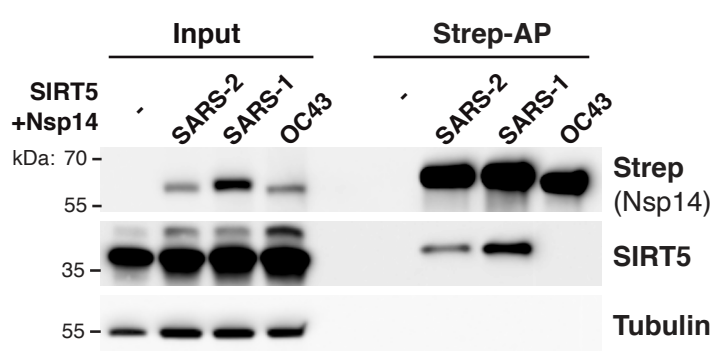

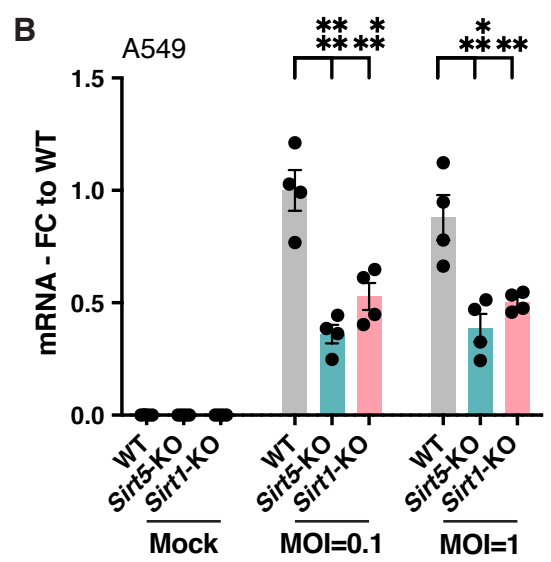

E

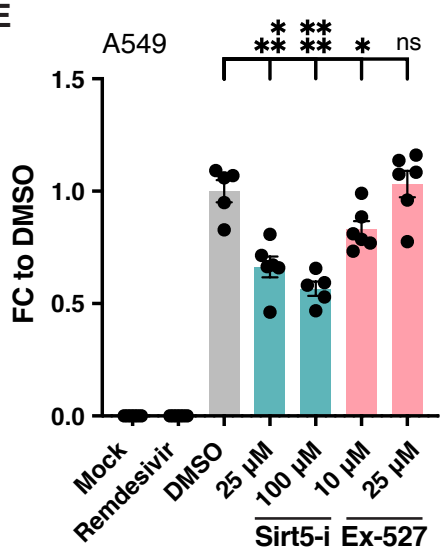

C

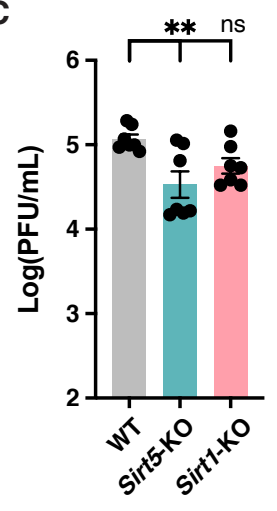

F

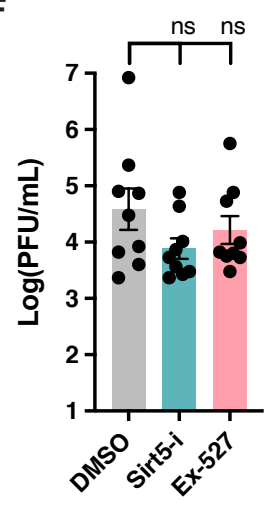

H

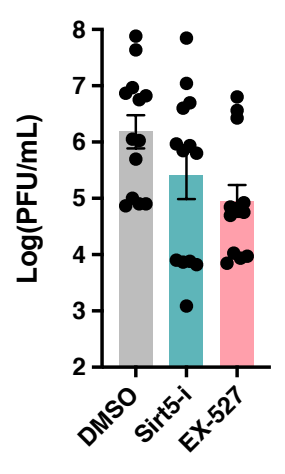

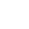

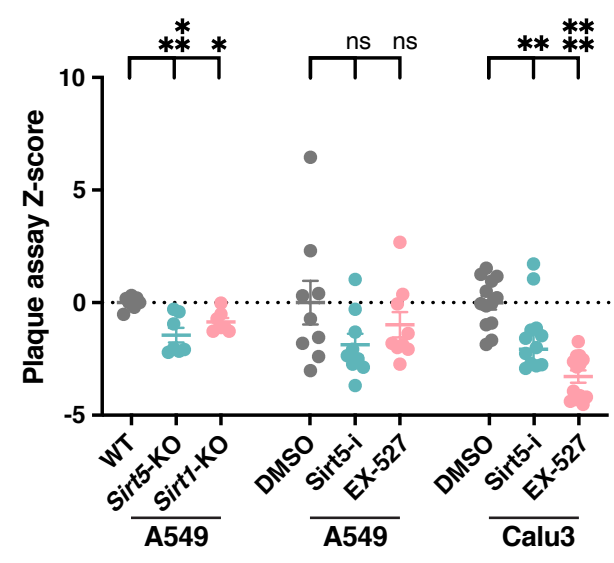

K

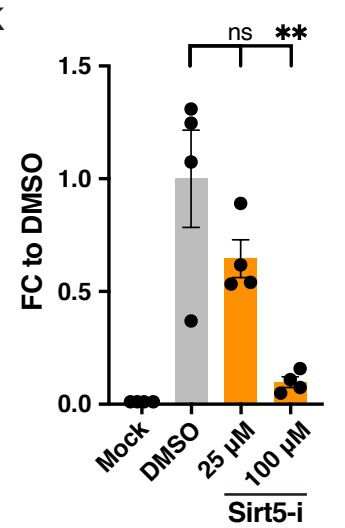


bioRxiv preprint doi: https://doi.org/10.1101/2022.01.04.474979; this version posted January 5, 2022. The copyright holder for this preprint (which was not certified by peer review) is the author/funder, who has granted bioRxiv a license to display the preprint in perpetuity. It is made available under aCC-BY-NC-ND 4.0 International license.

Walter et al., 2022 - SIRT5/Nsp14

\section{Figure 4: SIRT5 is a proviral factor}

A. Western blot showing the absence of SIRT5 and SIRT1 in Sirt5- and Sirt1-KO A549-ACE2 cells, after CRISPR knockout. B. Decrease in cell-associated viral mRNA levels in Sirt5- and Sirt1-KO cells infected with SARS-Cov-2 for 3 days at MOI=0.1 or MOI=1, as shown by RT-qPCR. Data show fold-changes compared to WT levels (at MOI=0.1). $\mathrm{n}=4$. C. Decrease in viral titers in Sirt5- and Sirt1-KO cells infected with SARS-Cov-2 for 3 days at $\mathrm{MOI}=1$, as shown by plaque assay. $n=6$. $\mathbf{D}$. Absence of cytotoxicity in A549 cells treated with Sirt5-i and Ex-527 inhibitor, as measured by flow cytometry. $n=4$. E. Decrease in cell-associated viral mRNA levels in A549-ACE2 cells infected with SARS-Cov-2 for 3 days at MOI=0.1, and treated with SIRT5 and SIRT1 inhibitors Sirt5-i and Ex-527, as shown by RT-qPCR. Data show fold-change compared to DMSO-treated levels. $n=6$. $F$. Decrease in viral titers in A549-ACE2 cells infected with SARS-Cov-2 for 3 days at MOI=0.1, and treated with SIRT5 and SIRT1 inhibitors Sirt5-i and Ex-527, as shown by plaque assay. $n=9$. $G / H$. Same as $E$. (with $n=4)$, and $F$. $(n=13)$, using Calu3 cells. I. Statistical analysis of plaque assay data to account for the batch effect between experiments performed on different days. A standardized Z-score was calculated and plotted, showing a significant reduction of viral titers in A549-ACE2 KO cells and Calu3. J. Strep-tag affinity-purification and western blot after transfection of Sirt5-KD HEK293T cells with SIRT5 and Nsp14-strep from different coronaviruses, showing that the interaction with SIRT5 is specific to SARS-like coronaviruses. K. Decrease in supernatant-associated viral mRNA levels in HCT-8 cells infected with HCoV-OC43 for 5 days at $\mathrm{MOI}=0.1$, and treated with SIRT5 inhibitor Sirt5-i, as shown by RT-qPCR. Data show fold-change compared to DMSO-treated levels. $n=4$. L. Decrease in viral titers in HCT-8 cells infected with HCoV-OC43 for 5 days at MOI=0.1, and treated with SIRT 5 inhibitors Sirt5-i, as shown by plaque assay. $n=4$.

Data show mean and standard error of the mean (SEM) between biological replicates. RT-qPCR results were internally normalized with Gapdh and Actin reference genes. Viral titers after plaque assay are expressed in log-transformed PFU (plaque-forming unit) per $\mathrm{mL}$ of supernatant. Asterisks summarize the results of statistical tests. ${ }^{*}: p<0.05,{ }^{* *}: p<0.01,{ }^{* * *}: p<0.001,{ }^{* * * *}: p<0.0001$. For RT-qPCR data and plaque assay Z-scores, we used one-way ANOVAs followed by Holm-Šidák multiple comparisons test. For plaque assays, we used one-way ANOVAs followed by Holm-Šidák multiple comparisons test on log-transformed data. 
decreased in A549-ACE2 Sirt5-KO cells ( $p=0.0005)$, as well as in Calu3 cells treated with Sirt5-i inhibitor ( $p=0.0032$ ). In A549-ACE2 cells treated with Sirt5-i, results did not reach statistical significance $(p=0.14)$. Altogether, we found that knocking out or inhibiting SIRT5 resulted in a decrease in SARS-CoV-2 levels.

To determine if the proviral role of SIRT5 could be explained by its interaction with Nsp14, we analyzed the role of SIRT5 during infection with human coronavirus HCOV-OC43, a distantly related beta-coronavirus. SIRT5 interacts with Nsp14 from SARS-CoV-2 and SARS-CoV, but not from MERS-CoV (2). Similarly, we observed by co-affinity purification in HEK293T cells that SIRT5 was not interacting with Nsp14 from HCoV-OC43 (Fig. 4J). This result further confirmed that SIRT5 interaction with Nsp14 is specific to SARS-like coronaviruses. Human colon adenocarcinoma cells HCT-8 were infected with HCoV-OC43 in presence of Sirt5-i inhibitor, and we observed a significant decrease in viral levels (Fig. 4K, L). At $100 \mu \mathrm{M}$, viral mRNA in the cell-culture supernatant and viral titers measured by plaque assay were both reduced 10 -fold ( $p=0.008$ and $p<0.0001$, respectively). This observation suggested that the role of SIRT5 during infection might be partially independent from its interaction with Nsp14.

In summary, our observations indicated that SIRT5 is a proviral factor necessary for coronaviruses replication and/or propagation. We obtained similar results with SIRT1. SARS-CoV-2 replication was significantly reduced in Sirt1-KO A549-ACE2 cells, with a twofold decrease in mRNA levels $(p=0.0002$ and $p=0.0011$ at $\mathrm{MOI}=0.1$ and 1 , respectively), and a twofold decrease in viral titers as well (Fig. 4A-C, I. $p=0.0145$ with plaque assay Z-score analysis). SIRT1 inhibitor Ex-527 had no effect in A549-ACE2 cells, but significantly reduced viral mRNA levels in Calu3 cells, with a fivefold reduction at $25 \mu \mathrm{M}(\mathrm{p}<0.0001$, Fig. 4D-H), and a strong reduction of viral titers as well ( $p<0.0001$, Fig. 4I). This showed that SIRT1 is also a proviral factor important for efficient SARS-CoV-2 infection. The reduction of viral levels was less pronounced and consistent than with SIRT5, and we focused the rest of our analysis on SIRT5.

\section{Sirt5 knockout cells express a higher basal level of viral restriction factors}

To gain insight into the role of SIRT5 during SARS-CoV-2 infection, we performed RNA-seq in A549-ACE2 cells, WT and Sirt5-KO, after 3 days of infection with SARS-CoV-2 (MOI=1).

Sequencing was done in four biological replicates for each condition. Principal-component analysis showed that samples separated well, based on knockout and infection status (Supplementary Fig. S2A). When comparing uninfected WT and Sirt5-KO cells, 1139 and 869 genes were significantly up- and downregulated, respectively ( $q$-value threshold $q=0.05$ ). Most of these changes were modest, and only 69 genes were up- or downregulated by more than twofold (Fig. 5A, left panel). Gene Ontology and pathway enrichment analysis showed that up-regulated genes were principally involved in metabolism and, in particular, protein catabolism processes in the lysosome, whereas down-regulated genes were involved in DNA replication and mitosis (Supplementary table S2). These findings were in line with reports showing that SIRT5 is implicated in autophagy and controls cell proliferation in cancer cells by targeting multiple metabolic enzymes $(31,32,38-41)$. In WT infected samples, around $5 \%$ 
bioRxiv preprint doi: https://doi.org/10.1101/2022.01.04.474979; this version posted January 5, 2022. The copyright holder for this preprint (which was not certified by peer review) is the author/funder, who has granted bioRxiv a license to display the preprint in perpetuity. It is made available under aCC-BY-NC-ND 4.0 International license.

Walter et al., 2022 - SIRT5/Nsp14

A

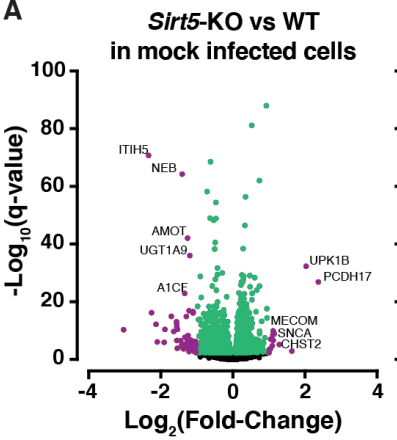

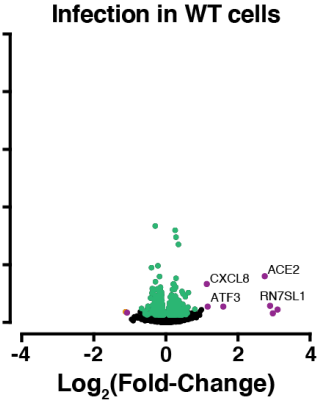

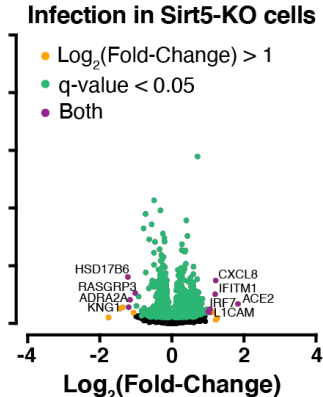

E

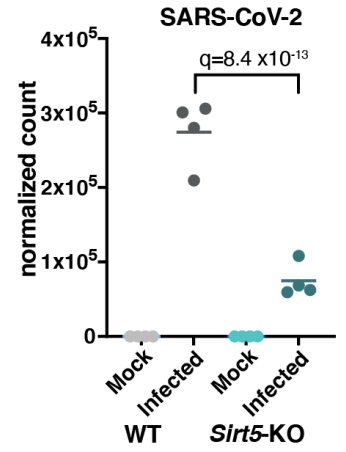

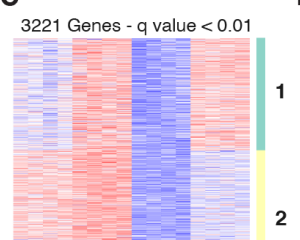

Cluster 1 (406 genes)
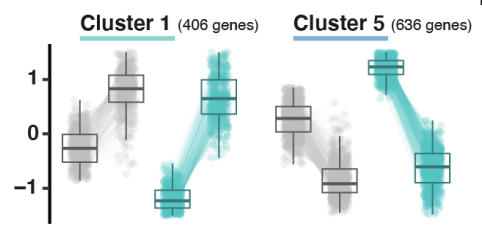

Cluster 2 (509 genes)
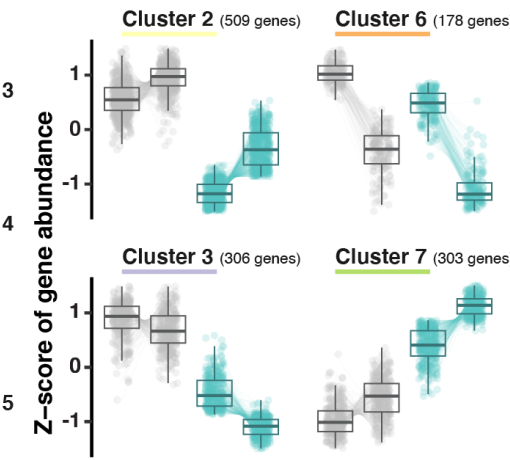

Cluster 7 (303 genes)

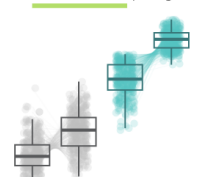

Cluster 4 (665 genes)

Cluster 8 (218 genes)
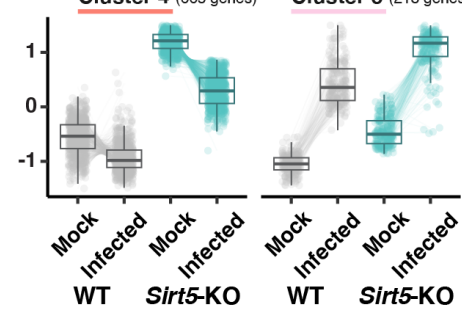

Enriched gene sets

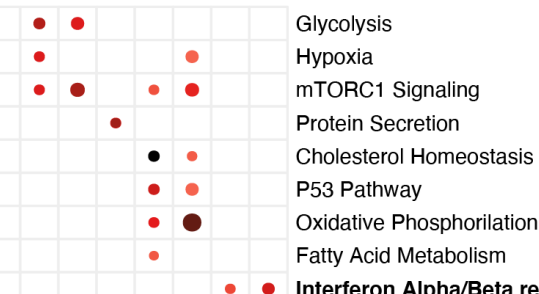

- Interferon Alpha/Beta response Coagulation

- Interferon Gamma response

- TNFa Signaling via NFkB Nonsense Mediated Decay Ribosomal RNA processing Metabolism of Amino Acids Cell cycle checkpoints Translation

Mitosis

DNA Replication

Signaling by Rho GTPases

Extracellular matrix organisation

Neutrophil Degranulation Antigen presentation by MHC class I

ER quality control compartment Cholesterol Biosynthesis

TCA Cycle Glucose Metabolism

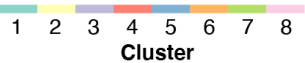

- $\log _{10}(\mathrm{p}$-value) Percent of cluster $\begin{array}{lllllllll}1 & 2 & 4 & 6 & 8 & 10 & 0 & 0.01 \\ 0 & 2 & 0 & 0.01\end{array}$

Figure 5: Sirt5-KO cells mount a stronger innate immune response.

RNA-seq analysis of WT and Sirt5-KO A549-ACE2 cells infected or mock-infected for 3 days with $\mathrm{SARS}-\mathrm{CoV}-2$ at $\mathrm{MOI}=1 . \mathrm{n}=4$. A. Volcano plots showing differentially expressed genes between the different conditions. Highlighted genes display a q-value $\mathrm{q}<0.05$ (green), log2 fold-change $>1$ (orange), or both (purple). Left panel: Sirt5-KO vs WT in mock-infected cells. Middle: Infected vs mock-infected WT cells. Right: Infected vs mock-infected Sirt5-KO cells. B. Normalized gene count of SARS-CoV- 2 . C-D. Unsupervised clustering of the 3221 genes differentially expressed between at least two of the four conditions $(\mathbf{q}<0.01)$. C: heatmap of normalized expression. D. Z-scores of differentially expressed genes as grouped by clustering. Colored lines represent quantification of an individual gene whereas solid black lines show the cluster Tukey boxplot. E. Enrichment analysis of biological gene sets in the identified gene clusters (C and $\mathrm{D})$. 
of total reads mapped to the SARS-CoV-2 genome, indicating substantial and successful viral replication. Despite this high level of viral expression, we observed a muted response to infection, at least in WT cells (Fig. 5A, middle panel). SARS-CoV-2 efficiently evades innate immune defense through multiple mechanisms, and this absence of a strong transcriptional response is characteristic of SARS-CoV-2 infection and has been documented in numerous studies (42-44). For example, we could not detect induction of IFN- $\alpha$, IFN- $\beta$, CXCL10, interleukin IL-6 or tumor necrosis factor (TNF). When comparing WT infected and WT mock-infected samples, 275 and 385 genes were significantly up- and down-regulated, respectively (threshold $q=0.05$ ), with only the chemokine CXCL8 (IL-8) and the transcription factor ATF3 being increased with a fold-change higher than 2 (Fig. 5A, middle). Gene Ontology and pathway enrichment analysis indicated that genes involved in response to virus infections were upregulated, with the two most upregulated pathways being the NOD-like and RIG-I-like receptors signaling pathways, which are implicated in the intracellular recognition of viruses (Supplementary table S2). Notably, other studies that reported a higher number of differentially expressed genes often had higher levels of viral infection, with a fraction of SARS-CoV-2 reads of $10-50 \%$, compared to $5 \%$ in this study. This might explain why the transcriptional response that we observed is comparatively smaller $(42,45)$.

We next analyzed the effect of Sirt5 knockout on SARS-CoV-2 infection. SARS-CoV-2 levels were almost fourfold less in Sirt5-KO infected cells than in WT infected cells, confirming that SIRT5 is a proviral factor $\left(q=8.4 \times 10^{-13}\right.$, Fig. 5B). We focused our analysis on the 3221 genes that were differentially expressed between at least two of the four conditions (threshold $q=0.01$ ). Hierarchical consensus clustering of the differentially expressed genes generated eight clusters, representing groups of genes that behaved similarly between the different sample conditions (Fig. 5C, D). Enrichment analysis of biological gene sets then allowed the identification of the cellular pathways over-represented in the identified clusters (Fig. 5E). For example, clusters 2 and 3 corresponded to genes with a lower expression in Sirt5-KD cells, independently of the infection status, and pathways linked to the cell cycle and cellular metabolism were significantly enriched in these clusters. In most of the clusters (clusters 2-6), pathways linked to cellular metabolism were significantly enriched, highlighting that SIRT5 is an important metabolic enzyme. Clusters 7 and 8 were particularly interesting. They represent genes that are expressed at a higher basal level in Sirt5-KO cells, and whose expression is further increased during infection (Fig. 5C, D). Strikingly, pathways linked to innate immunity, such as type I and II interferon and NFKB signaling, were significantly enriched in these clusters (Fig. 5E). Genes in clusters 7 and 8 are up-regulated in Sirt5-KO cells, even in uninfected cells, which suggested that Sirt5-KO cells had a higher basal level of innate immunity markers and could mount a stronger immune response.

We thus investigated whether innate immunity pathways were up-regulated in Sirt5-KO cells, even without a viral infection. In mock-infected cells, the Gene Ontology term "Innate Immune Response" was significantly enriched in Sirt5-KO samples ( $q=0.0071$, Supplementary table S3). Type-I interferon responses are one of the most important lines of defense against viruses. We analyzed the expression of known Type-I interferon-stimulated 
genes, as well as of other genes broadly linked to innate immune responses and present in clusters 7 and 8 (Fig. 6). As could be expected, most of these genes were upregulated in infected cells. Strikingly, many of these genes, which are normally involved in the response to pathogens, were also upregulated in Sirt5-KO cells in absence of infection, with 39 out of the 71 selected genes being significantly upregulated in mock-infected Sirt5-KO cells $(q<0.05)$. Many of these genes were expressed at similar levels between infected WT cells and mock-infected Sirt5-KO cells, and were further increased in infected Sirt5-KO cells (Fig. 6). This included cytokines (IL33, CXCL5, and CSF1), the transmembrane restriction factors IFITM2 and 3, members of the complement system (C1S, C1R and C3), the MHC class I subunit B2B, and other interferon-stimulated genes or restriction factors (e.g., TRIM22, STAT3, MMP7, LCN2, MUC5AC, SLFN5, NT5E, CAST and SNCA) (46-52). The upregulation between WT and Sirt5-KO in mock-infected cells was modest but statistically significant, ranging from 20 to $50 \%$, and was further increased up to twofold in infected Sirt5-KO cells (Fig. 6, Supplementary Fig. S2A). These results suggest that Sirt5-KO cells express a higher basal level of numerous restriction factors and mount a stronger antiviral response, which could explain why SARS-CoV-2 levels are decreased in absence of SIRT5.

\section{Discussion}

In this study, we investigated the role of SIRT5 during SARS-CoV-2 infection. Our results show that SIRT5 stably interacts with the non-structural viral protein Nsp14, and that this interaction is independent of Nsp10. Interestingly, we found that the catalytic activity of SIRT5 was necessary for the interaction, as several SIRT 5 catalytic mutants could not bind to Nsp14, and the interaction was blocked by specific SIRT5 inhibitors. SIRT5 is the main cellular desuccinylase, demalonylase, and deglutarylase, but we could not detect these lysine modifications on Nsp14 protein, suggesting that Nsp14 is not directly modified by SIRT5. We further showed that SIRT5 is a proviral factor and that SARS-CoV-2 levels decrease when SIRT5 is deleted or inhibited in cell-culture experiments. We observed that Sirt5-KO cells express innate immunity markers at a higher basal level and mount a stronger antiviral response, which might explain the decrease in viral levels. Taken together, our study uncovered a novel and unexpected role for SIRT5 during SARS-CoV-2 infection.

The interaction between SIRT5 and Nsp14 is intriguing. Mutating the SIRT5 catalytic domain or treating cells with a SIRT5 inhibitor blocked the interaction (Fig. 2B, C), and the strength of the interaction appeared to be modulated by cellular NAD levels (Fig. 2D). NAD is necessary for SIRT5 activity, and these results highlight that SIRT5 catalytic activity or at the very least a functional catalytic domain is necessary to interact with Nsp14. SIRT5 binds to some of its targets in co-purification experiments, such as with MAVS, SHMT2, or PKM2 (30-32). However, in these examples, SIRT5 was also desuccinylating the target proteins. Here we found no lysine modifications on Nsp14, either by mass spectrometry or immunoblotting. Our experiments might not have been sensitive enough to detect it, but our mass spectrometry data had very high purity, a coverage close to $90 \%$ on Nsp14, and our pipeline 
bioRxiv preprint doi: https://doi.org/10.1101/2022.01.04.474979; this version posted January $5,2022$. The copyright holder for this preprint (which was not certified by peer review) is the author/funder, who has granted bioRxiv a license to display the preprint in perpetuity. It is made available under aCC-BY-NC-ND 4.0 International license.

Walter et al., 2022 - SIRT5/Nsp14

A
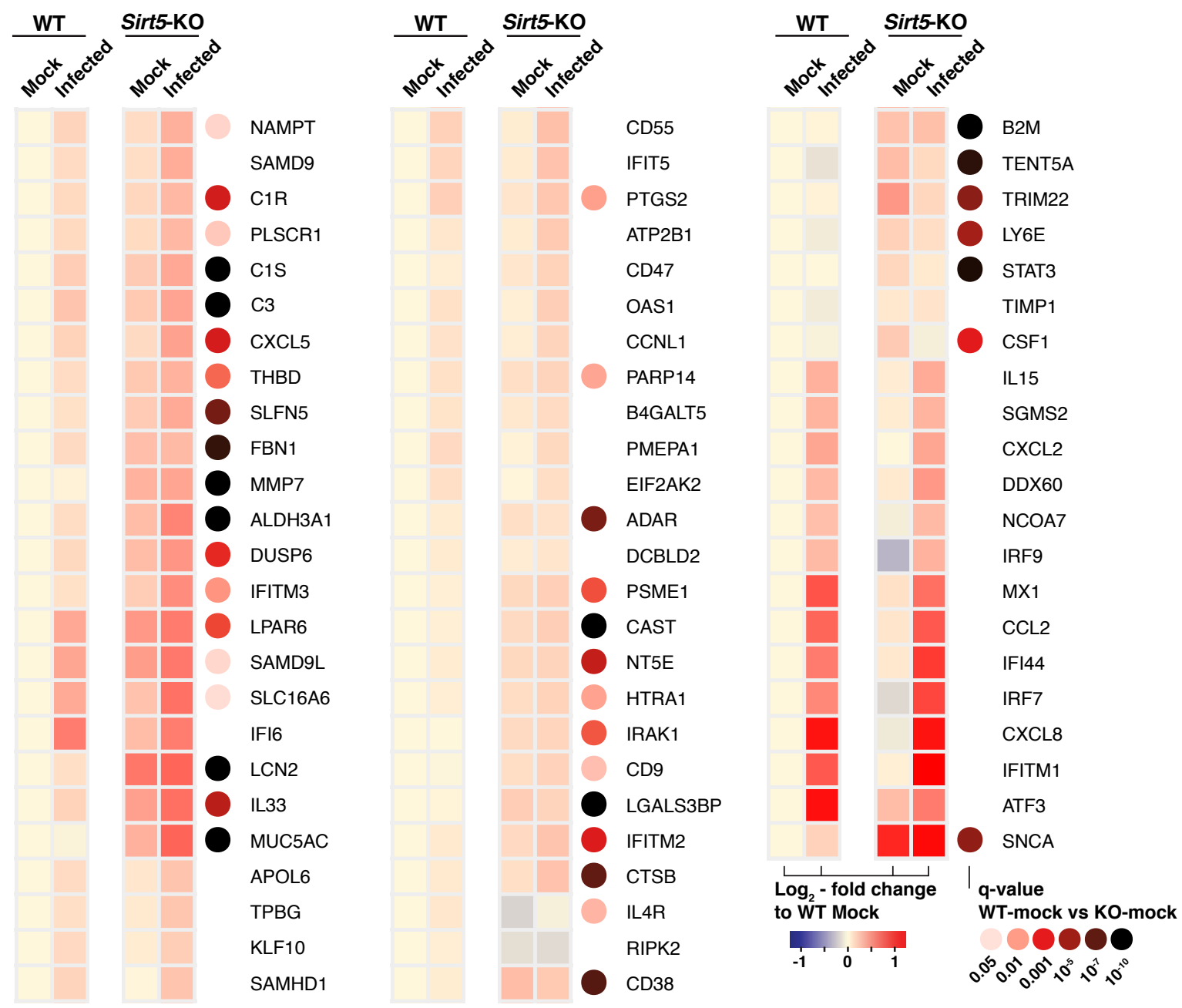

B

Model 1:

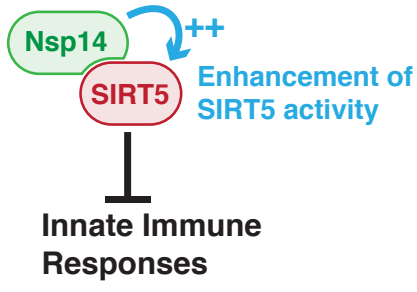

Model 2:

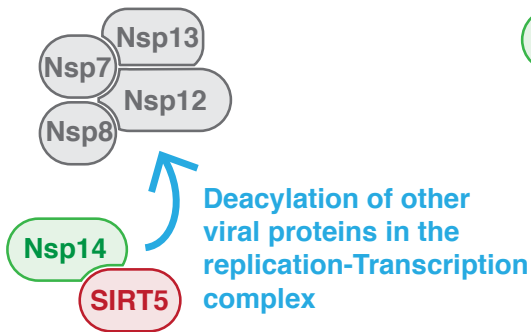

Model 3:

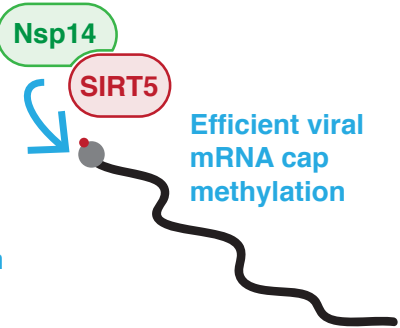

Figure 6: Sirt5-KO cells express higher basal level of viral restriction factors.

A. Expression heatmap of interferon-stimulated genes and other restriction factors, showing that mock-infected Sirt5-KO cells express higher basal levels of restriction factors, and that antiviral responses are stronger in Sirt5-KO cells. Data show mean log2 fold-change, compared to mock-infected WT, and the q-value between mock-infected WT and Sirt5-KO cells. Only genes differentially expressed between at least two conditions were included in the analysis $(q<0.01)$. B. Hypotheses for the role of the SIRT5/Nsp14 interaction during SARS-CoV-2 infection. In model 1, Nsp14 could enhance SIRT5 activity, which would decrease innate immune responses and favor viral replication. In model 2, Nsp14 could redirect SIRT5 to novel targets, potentially in the replication-transcription complex, where SIRT5 could deacylate other viral proteins. In model 3, the Nsp14/SIRT5 complex could be primarily involved in mRNA cap methylation. Absence or inhibition of SIRT5 would lead to incomplete cap methylation and stronger immune recognition of viral mRNA. 
routinely detects such modifications. Besides, SIRT5 is the only known desuccinylase, demalonylase, and deglutarylase, and these experiments were performed in Sirt5-KD cells, which would have enriched the presence of these lysine modifications if they were present. The unusual nature of the interaction might explain why we were unable to reconstitute the complex in vitro with proteins purified in E. coli, and why our enzymatic assays failed to uncover a clear molecular function. Interestingly, an interaction of a similar nature has been described between SIRT1 and the HIV viral protein Tat $(53,54)$. Tat interacted with the SIRT1 catalytic domain and mutation of conserved residues disrupted the interaction. In this case however, Tat was deacetylated by SIRT1 and blocked SIRT1 activity. Here, we could not determine if SIRT5 activity was altered by Nsp14, and global succinylation and malonylation levels appeared unaffected as well (Fig. 2E). Further studies will be necessary to understand the nature of the interaction and its function. They could reveal a novel interaction mechanism, in which an enzyme binds to a partner through its catalytic domain, which has rarely been observed.

The role of SIRT5 and other sirtuins in disease is unclear. SIRT5, by modulating key metabolic enzymes, could be involved in cancer (31, 32, 38-41). Sirt5-KO mice display no obvious phenotype and mount strong innate immune responses against several bacterial infections (55). The role of SIRT5 and other sirtuins during viral infection is understudied and likely depends on the pathogen. Knocking-out SIRT5 enhances the replication of several DNA viruses, such as herpes simplex 1 , human cytomegalovirus and adenovirus type 5 , and the same study reported a potential increase in influenza replication, albeit non-significantly (56). By contrast, here we showed that inhibition or deletion of SIRT5 led to a decrease of viral levels with two distinct coronaviruses, SARS-CoV-2 and HCoV-OC43, indicating that SIRT5 is a proviral factor in this context (Fig. 4). A recent study showed that replication of vesicular stomatitis virus (VSV) and Sendai virus (SeV), two negative-strand RNA viruses, was also diminished in absence of SIRT5 (30). Whether SIRT5 broadly acts as a restriction factor against DNA viruses and as a proviral factor against RNA viruses will be an interesting hypothesis to investigate in the future.

The decrease of SARS-CoV-2 levels in Sirt5-KO cells correlated with elevated basal levels of numerous viral restriction factors, even in mock-infected cells (Fig. 6). This upregulation was modest but highly significant and may account for why SARS-CoV-2 propagated slower in Sirt5-KO cells. Several hypotheses could explain this elevation of innate defenses. SIRT5 has been directly implicated in the RIG-I/MAVS pathway, a critical component of the innate sensing of RNA virus (30). Recognition of cytosolic RNA molecules by RIG-I-like receptors, including RIG-I and MDA5, causes recruitment of MAVS, which forms large aggregates on the surface of mitochondria, ultimately leading to type I IFN production (57). MAVS was succinylated upon viral challenge with VSV and SeV, and desuccinylation of MAVS by SIRT5 diminished MAVS aggregation, resulting in lower interferon activation. By preventing MAVS aggregation, SIRT5 therefore enhances viral replication, at least with VSV and SeV (30). However, SARS-CoV-2 efficiently counteracts MAVS activation of innate defense. The coronavirus proteins $\mathrm{M}$, Orf9b and Orf3b directly antagonize MAVS aggregation or 
downstream signaling (58-60), and MAVS knockout has no effect on SARS-CoV-2 replication (43). This suggests that the desuccinylation of MAVS by SIRT5 is not the main mechanism for explaining the decrease of viral levels in Sirt5-KO cells. Nonetheless, sustained absence of SIRT5 could lead to a higher basal level of MAVS aggregation that, in turn, could cause the higher level of restriction factors that we observed. Other mechanisms could be involved. For example, SIRT5 is involved in the detoxification of reactive oxygen species (ROS) and attenuates cellular ROS levels (27). Elevated ROS levels activate innate immune responses, and the absence of SIRT5 could cause the activation of innate immune responses through this pathway (61). Furthermore, SIRT5 regulates proteins involved in glycolysis, the TCA cycle, and fatty acid oxidation. These pathways were impaired without SIRT5, as well as mTOR signaling (Fig. 5E). mTOR and the cellular sensing of energy and nutrient levels can directly influence immune activity (62), highlighting another pathway that could lead to immune activation in absence of SIRT5.

The potential link between our two key findings, namely the Nsp14/SIRT5 interaction and the proviral role of SIRT5, will be the subject of further investigation. Several hypotheses can be considered (Fig. 6B). First, Nsp14 might work by enhancing SIRT5 activity, which would favor viral replication by dampening innate immune responses. In this model, increased deacylation of cellular targets of SIRT5 such as MAVS could result in a weaker immune response and favor viral replication. Second, Nsp14 could redirect SIRT5 toward novel targets, for example other viral proteins. Nsp14 is part of the viral replication-transcription complex, and SIRT5 could be involved in the deacylation of other members of the complex such as Nsp7, Nsp8, Nsp12 or Nsp13. Third, we observed that SIRT5 and Nsp10 were part of separate complexes, and that Nsp14 MTase activity increased slightly in presence of SIRT5 (Fig. 3G). SIRT5 and Nsp10 could be separately involved in the two enzymatic activities of Nsp14, with the Nsp14/SIRT5 complex primarily responsible for viral mRNA Cap methylation. Absence of SIRT5 would result in a defect in cap methylation, more efficient immune recognition of viral RNA, and a stronger immune response, as we observed.

To summarize, further studies will be necessary to elucidate how SIRT5 enhances SARS-CoV-2 replication, and how the interaction with Nsp14 plays in this context. Potent inhibitors of SIRT5 are in development, and SIRT5 is a potential target against cancer $(34,40$, 63). Our manuscript highlights that SIRT5 is a potential pharmaceutical target that could help against viral infections as well, and SARS-CoV-2 in particular. Currently, very few antiviral drugs are approved. Inhibiting SIRT5 will probably never represent a first line of defense, but it could be used in combination with drugs that directly target viral enzymes, leading to novel therapeutic regimens against COVID-19. 


\section{Materials and Methods}

\section{Mammalian cell lines and culture conditions}

All cell lines were maintained at $37^{\circ} \mathrm{C}$ in a $5 \% \mathrm{CO}_{2}$ humidified incubator. Cells were frequently tested for mycoplasma contamination and consistently tested negative.

HEK293T cells were obtained from ATCC (Cat. CRL-11268) and maintained in high glucose Dulbecco's minimal Eagle's medium (DMEM) with 10\% fetal bovine serum (FBS)

(Sigma-Aldrich, USA) and $100 \mu \mathrm{m} / \mathrm{L}$ penicillin-streptomycin (Corning, USA). Calu3 cells were obtained from ATCC (Cat. HTB-55) and cultured in AdvancedMEM (Gibco, USA), supplemented with $2.5 \%$ FBS (GeminiBio, USA), $1 \%$ GlutaMax, and $100 \mu \mathrm{m} / \mathrm{L}$ penicillin-streptomycin. Wild-type A549 cells were purchased from ATCC (Cat. CCL-185). A549 cells stably expressing ACE2 (A549-ACE2) were a gift from 0. Schwartz (Pasteur Institute, Paris). A549-ACE2 cells were cultured in DMEM, supplemented with $10 \%$ FBS (GeminiBio, USA) and blasticidin (20 $\mathrm{gg} / \mathrm{ml}$ ) (Sigma-Aldrich, USA). Short-terminal repeat (STR) analysis by the Berkeley Cell Culture Facility on 17 July 2020 authenticated these as A549 cells with $100 \%$ probability. Vero-E6 cells were obtained from ATCC (Cat. CRL-1586) and cultured in DMEM (Corning, USA), supplemented with 10\% FBS (GeminiBio, USA), 1\% glutamine (Corning), and $100 \mu \mathrm{m} / \mathrm{L}$ penicillin-streptomycin (Corning). HCT-8 cells (ATCC Cat. CCL-244) were cultured in DMEM with 10\% FBS and penicillin-streptomycin.

Small-molecule inhibitors used in the study are listed in Supplementary Table S3.

\section{Plasmids}

Plasmids expressing GFP and Nsp14 proteins (from SARS-CoV-2, SARS-CoV and HCoV-OC43) with a C-terminus strep tag were a gift from Nevan Krogan (1, 2), and are also available on Addgene (pLVX-EF1alpha-SARS-CoV-2-nsp14-2xStrep-IRES-Puro, Addgene \#141380). Nsp10-Flag plasmid was a gift from the Ott lab.

Mammalian expression plasmids for SIRT5 and SIRT5-H158Y with a myc-his tag in a pCDNA 3.1 vector were available in the Verdin lab (26). Y102F, R105M, Q140A and I142A mutants were generated by Q5 site-directed mutagenesis (NEB, USA).

\section{Generation of HEK293T Sirt5 knockdown and A549-ACE2 Sirt5 knockout cell lines}

The Sirt5 knockdown (Sirt5-KD) cell line was generated using CRISPR interference in HEK293T cells. First, we generated a stable cell line expressing dCas9-KRAB-MeCP2. Early passage HEK293T cells were transfected with $1.5 \mu \mathrm{g}$ of dCas9-KRAB-MeCP2 repressor plasmid (Addgene \#110824) and $0.5 \mu \mathrm{g}$ of Piggyback Transposase (gift from Maxim Greenberg), using PEI 25K transfection reagent (Polysciences Inc, Cat. 23966-1), according to the manufacturer's instructions. Cells stably expressing dCas9-KRAB-MeCP2 were selected with Blasticidin (Invivogen, USA) for 10 days before generating Sirt5-KD cells. Second, sequences for sgRNA against human Sirt5 (5'- GGCGCTCCGGACCTGAGCCA-3') or non-targeting sgRNA (5'-GCTGCATGGGGCGCGAATCA-3') were obtained from Horlbeck et al. (64) and cloned into Addgene \#84832 by annealing and ligation using T4 ligase. Plasmids were validated by Sanger sequencing (Elim Biopharmaceuticals). Lentiviruses expressing the 
gRNAs were produced in HEK293T cells by standard protocols (65). HEK293T cells expressing dCas9-KRAB-MeCP2 were infected with lentiviruses in medium containing 1 $\mu \mathrm{g} / \mathrm{ml}$ polybrene and $30 \%$ lentivirus-containing supernatant $(\mathrm{v} / \mathrm{v})$. Cells were then selected with Blasticidin at $5 \mu \mathrm{g} / \mathrm{mL}$ and Puromycin ( $1 \mu \mathrm{g} / \mathrm{mL}$, Invivogen, USA) to select for cells stably expressing both dCas 9 and the sgRNAs. Stable knockdowns were validated by western blot.

The Sirt5 knockout (Sirt5-KO) cell line was generated using CRISPR-Cas9 editing in A549-ACE2 cells. sgRNAs were commercially designed by Synthego (Redwood, California, USA) and are designed to work cooperatively to generate small-fragment deletions in early exons causing knockout (Supplementary Table S2). We combined $10 \mathrm{pmol}$ of Streptococcus pyogenes NLS-Sp.Cas9-NLS (SpCas9) nuclease (Aldevron, USA, Cat. 9212) with 30 pmol of total synthetic sgRNA (10 pmol each sgRNA) to form ribonucleoproteins (RNPs) in $20 \mu \mathrm{L}$ of total volume with SE Buffer for A549-ACE2 cells. The RNP assembly reaction was mixed by pipetting up and down and incubated at room temperature for 10 minutes. Cells were resuspended in transfection buffer, according to cell type, added to the preformed RNP solution, and gently mixed. Nucleofections were performed on a Lonza nucleofector system (Lonza, Switzerland, Cat. AAU-1001), using program CM-120 for A549-ACE2 cells. Cells were grown for several passages and genotyped by PCR and Sanger sequencing to confirm efficient editing. Absence of SIRT5 was confirmed by western blot.

\section{Transfection, Strep affinity purification, and Flag immunoprecipitation in HEK-293T cells}

HEK-293T cells were plated in six-well plates or 10-cm dishes. After 24 hours, cells were transfected using polyethylenimine (PEI). Nsp14 is cytotoxic, and we used $0.5 \mu \mathrm{g}$ of Nsp14-strep plasmid for a six-well plate and $4 \mu \mathrm{g}$ for a $10-\mathrm{cm}$ dish. Other co-transfecting plasmids, such as pcDNA-SIRT5, were used at the same concentration except when specifically mentioned. The total amount of plasmid was normalized using empty vectors when necessary. Plasmids were complexed with PEI in Opti-MEM medium (Thermofisher) at a 1:3 ratio, and the mixture was deposited onto cells dropwise. After 48 hours, cells were washed once with PBS, scraped off the plate by thorough pipetting, pelleted by centrifugation at $200 \mathrm{~g}$ and $4{ }^{\circ} \mathrm{C}$ for 3 minutes, and frozen at $-80^{\circ} \mathrm{C}$.

Affinity purification followed the methods of Gordon et al. 2020 (1). Frozen cell pellets were thawed on ice and resuspended in $0.5 \mathrm{ml}$ of lysis buffer (IP buffer: $50 \mathrm{mM}$ Tris-HCl, $\mathrm{pH} 7.4$, $150 \mathrm{mM} \mathrm{NaCl}$, and $1 \mathrm{mM}$ EDTA), supplemented with $0.5 \%$ Nonidet P40 substitute (NP40; Fluka Analytical) and cOmplete mini EDTA-free protease and PhosSTOP phosphatase inhibitor cocktails (Roche)). Samples were frozen on dry ice for 10-20 minutes and partially thawed at $37^{\circ} \mathrm{C}$ before incubation on a tube rotator for 30 minutes at $4{ }^{\circ} \mathrm{C}$, and centrifugation $\left(13,000 \mathrm{~g}, 4^{\circ} \mathrm{C}, 15\right.$ minutes) to pellet debris. $30 \mu \mathrm{L}$ of "input" was saved and frozen at $-80^{\circ} \mathrm{C}$. $20 \mu \mathrm{L}$ of MagStrep 'type3' beads (IBA Lifesciences) were equilibrated twice with $1 \mathrm{~mL}$ of wash buffer (IP buffer supplemented with $0.05 \%$ NP40) and incubated with 0.5 $\mathrm{ml}$ of lysate for $2 \mathrm{~h}$ at $4{ }^{\circ} \mathrm{C}$ on a tube rotator. Tubes were placed on a magnetic rack, and beads were washed three times with $1 \mathrm{ml}$ of wash buffer, and samples were shortly vortexed 
between washes. Bound proteins were eluted for 30 minutes using $30 \mu \mathrm{L}$ of BXT elution buffer (IBA Lifesciences) with constant shaking at room temperature. Tubes were placed back on the magnetic rack, and the eluate was recovered and frozen at $-80^{\circ} \mathrm{C}$.

Flag-immunoprecipitation was performed the same way using Anti-FLAG M2 Magnetic Beads (Sigma-Aldrich M8823) and elution was done using 3x-Flag peptide (Sigma-Aldrich F4799) at a concentration of $3 \mu \mathrm{g} / 50 \mu \mathrm{L}$ in IP buffer. When performing side by side Strep-affinity purification and Flag-immunoprecipitation, the same frozen cell sample was divided in two after lysis.

\section{Western blot}

Western blot was performed using standard protocols. Briefly, protein lysate was mixed with $4 \mathrm{x}$ Laemmli sample buffer containing DTT and boiled for 5 minutes at $95^{\circ} \mathrm{C}$. Proteins were separated on a precast $4-20 \%$ gradient gel (Biorad, USA) and transferred on a nitrocellulose membrane using a semi-dry Trans-Blot Turbo Transfer System and Trans-Blot Turbo Transfer Buffer (Biorad, USA). Membranes were blocked for 1 hour with $5 \%$ milk in TBST (Tris-buffered saline and Tween 20) buffer, rinsed, and incubated overnight at $4{ }^{\circ} \mathrm{C}$ with primary antibodies in $2 \%$ BSA in TBST. Membranes were washed three times with TBST and incubated for 2 hours at room temperature with horseradish peroxidase-linked secondary antibody. The chemiluminescent signal was revealed with SuperSignal ${ }^{\mathrm{TM}}$ West Pico PLUS Substrate (ThermoFisher, USA) and imaged with an Azure 600 Imaging system (Azure Biosystem, USA). Antibodies are listed in Supplementary Table S3.

\section{Cellular Thermal Shift Assay (CETSA)}

CETSA was performed as described (66). Shortly, HEK-293T cells in six wells were transfected with plasmids expressing Nsp14-strep and/or SIRT5. After 48 hours, cells were harvested, washed with PBS, and resuspended in PBS supplemented with EDTA-free complete protease inhibitor cocktail (Roche). Intact cells were divided into 100- $\mu$ l aliquots and heated individually at different temperatures for 3 minutes in a PCR machine (Biorad), followed by cooling for 2 minutes at room temperature. Cell suspensions were freeze-thawed three times with liquid nitrogen, and the soluble fraction was separated from the cell debris by centrifugation at $20,000 \times g$ for 20 minutes at $4{ }^{\circ} \mathrm{C}$. Supernatants containing soluble proteins were transferred to new microcentrifuge tubes and analyzed by western blot.

\section{Immunofluorescence}

A549 cells plated in an eight-well chamber slide (Nunc Lab-Tek II, Thermo Fisher) were transfected with $500 \mathrm{ng}$ of nsp14-strep plasmid encoding nsp14-strep using Lipofectamine 3000 (Thermo Fisher). The next day, cells were fixed in PBS-buffered $4 \%$ formaldehyde at room temperature. After 15 minutes, cells were briefly rinsed with PBS once and incubated in permeabilization buffer ( $0.1 \%$ Triton X-100 in PBS). After 15 additional minutes, cells were incubated in blocking buffer (permeabilization buffer supplemented with $1 \%$ BSA), and further incubated in blocking buffer containing anti-Strep mouse antibody (1:1000 dilution), 
and anti-Sirt5 rabbit antibody (1:1000 dilution). The next day, the cells were washed with PBS three times and incubated in the blocking buffer containing anti-mouse IgG donkey antibody conjugated with Alexa 488 (1:500 dilution, Thermo Fisher), anti-rabbit IgG donkey antibody conjugated with Alexa 555 (1:500 dilution, Thermo Fisher), and for counter-staining, DAPI (1 $\mu \mathrm{g} / \mathrm{ml}$, Sigma Aldrich) and Phalloidin conjugated with Alexa 647 (1: 1000 dilution, Abcam). After 30 minutes, the cells were washed with PBS three times, and mounted in prolong gold anti-fade (Thermo Fisher), followed by curing overnight. The cells were visualized using a confocal fluorescence microscope (LSM 700, Carl Zeiss) at $63 \mathrm{X}$ magnification, imaged, and analyzed using ZEN imaging software (blue edition, Ver 3.4, Carl Zeiss). Antibodies used are given in Supplementary Table S2.

\section{Protein purification and enzymatic assays}

Nsp10 and Nsp14 proteins from the Wuhan strain of SARS-CoV-2 (NC_045512.2) were codon-optimized, ordered as Gblocks (IDT), and cloned into a pVFT1S expression vector using a HiFi DNA Assembly kit (NEB). Both nsp10 and nsp14 contained an $\mathrm{N}$-terminal $6 \mathrm{x}$-His tag, followed by a TEV cleavage site. E. coli BL21*(DE3) cells (Invitrogen, USA) were transformed with the nsp10 and nsp14 expression vectors and grown in LB medium containing kanamycin. Cells were induced at an $\mathrm{OD}_{600}$ of $\sim 0.8$ with $0.5 \mathrm{mM}$ IPTG for 16 hours at $16^{\circ} \mathrm{C}$. Nsp10 pellets were stored at $-20^{\circ} \mathrm{C}$, and nsp 14 pellets were flash-frozen with liquid nitrogen and stored at $-80^{\circ} \mathrm{C}$ until use. For nsp $10 / 14$ copurification, nsp 10 pellets from $1 \mathrm{~L}$ of cells and nsp14 pellets from $6 \mathrm{~L}$ of cells were resuspended in lysis buffer ( $50 \mathrm{mM}$ HEPES, $\mathrm{pH}$ 7.5, $300 \mathrm{mM} \mathrm{NaCl}, 5 \mathrm{mM} \mathrm{MgSO}_{4}, 30 \mathrm{mM}$ imidazole, and 1\% NP-40) and combined. For nsp14 purification, pellets were resuspended in lysis buffer. The pellets were lysed using sonication and clarified using centrifugation at $14,500 \mathrm{rpm}$ for 40 minutes at $4^{\circ} \mathrm{C}$. The supernatant was loaded onto a HisTrap HP column (GE Healthcare, USA). Proteins were purified by fast protein liquid chromatography and washed using two column volumes of Ni Buffer A (50 mM HEPES, pH 7.5, $300 \mathrm{mM} \mathrm{NaCl}^{5} \mathrm{mM} \mathrm{MgSO}_{4}$, and $30 \mathrm{mM}$ imidazole). For nsp10/14 copurification only, an additional wash was done over five column volumes using a buffer of $50 \mathrm{mM}$ HEPES, $\mathrm{pH}$ 7.5, $300 \mathrm{mM} \mathrm{NaCl}, 5 \mathrm{mM} \mathrm{MgSO}_{4}$, and $60 \mathrm{mM}$ imidazole. Proteins were eluted using $50 \mathrm{mM}$ HEPES, $\mathrm{pH} 7.5,300 \mathrm{mM} \mathrm{NaCl}, 5 \mathrm{mM} \mathrm{MgSO}_{4}$, and $300 \mathrm{mM}$ imidazole. The elution was then concentrated and purified further using a Superdex 200 column (GE) and a buffer of $10 \mathrm{mM}$ HEPES, $\mathrm{pH} 7.5,150 \mathrm{mM} \mathrm{NaCl}$, and 10\% glycerol. The purified protein was then concentrated, flash-frozen using liquid nitrogen, and stored at $-80^{\circ} \mathrm{C}$.

SIRT5 purified protein was obtained commercially (BPS Bioscience, USA, Cat. 50016). In vitro desuccinylation assays were performed using Fluorogenic SIRT5 Assay Kit (BPS Bioscience, USA, Cat. 50085), following the manufacturer's instructions. Methyltransferase assays were performed using MTase-Glo ${ }^{\mathrm{TM}}$ Methyltransferase Assay (Promega, USA), following the manufacturer's instructions. Nsp14 and SIRT5 recombinant proteins were first incubated together in the reaction buffer, with a ratio of 1:1 corresponding to $100 \mathrm{nM}$ of each protein. Desuccinylation assays were performed in a reaction buffer $(25 \mathrm{mM}$ Tris $/ \mathrm{HCl}, \mathrm{pH} 8.0,137$ $\mathrm{mM} \mathrm{NaCl}, 2.7 \mathrm{mM} \mathrm{KCl}, 1 \mathrm{mM} \mathrm{MgCl}$, and $0.1 \mathrm{mg} / \mathrm{ml} \mathrm{BSA}$ ) with $0.5 \mathrm{mM} \mathrm{NAD+} \mathrm{for} 30$ minutes at $37^{\circ} \mathrm{C}$. Methyltransferase assays were performed in reaction buffer (50 mM Hepes, $\mathrm{pH} 7.0$, 
$6 \mathrm{mM} \mathrm{KCl}, 2 \mathrm{mM} \mathrm{DTT}, 1 \mathrm{mM} \mathrm{MgCl}$, and $0.1 \mathrm{mg} / \mathrm{ml} \mathrm{BSA}$ ) in presence of $0.1 \mathrm{mM} \mathrm{NAD+}$ and 10 $\mu \mathrm{M}$ SAM.

\section{Sample Preparation for Proteomic Analysis}

HEK-293T Sirt5-KD cells were transfected with plasmids expressing Nsp14-strep in the presence or absence of SIRT5 with 3 biological replicates for each condition. Nsp14-strep and bound proteins were purified by affinity purification as described above, and eluted in 35 $\mu \mathrm{L}$ of elution buffer (100 mM Tris pH 7.4; $150 \mathrm{mM} \mathrm{NaCl} ; 1 \mathrm{mM}$ EDTA; $50 \mathrm{mM}$ biotin). Each sample was subjected to a lysis buffer containing $5 \%$ SDS and $50 \mathrm{mM}$ triethylammonium bicarbonate (TEAB) for proteomics sample preparation.

The samples enriched for NSP14 were reduced with $20 \mathrm{mM}$ dithiothreitol (DTT) in $50 \mathrm{mM}$ TEAB buffer at $50{ }^{\circ} \mathrm{C}$ for 10 minutes, left to cool at room temperature for 10 minutes, and alkylated with $40 \mathrm{mM}$ iodoacetamide (IAA) in $50 \mathrm{mM}$ TEAB buffer in the dark at room temperature for 30 minutes. Samples were acidified with a final concentration of $1.2 \%$ phosphoric acid. Subsequently, 90\% methanol in 100 mM TEAB was added. The entire sample volume was spun through the micro S-Trap columns (Protifi) to bind the proteins to the S-Trap column. The S-Trap columns were washed again with $90 \%$ methanol in $100 \mathrm{mM}$ TEAB. S-Trap columns were placed in a clean elution tube and incubated with trypsin digestion buffer ( $50 \mathrm{mM}$ TEAB, $\mathrm{pH} \sim 8$ ) at a 1:25 ratio (protease:protein, wt:wt) for 1 hour at $47^{\circ} \mathrm{C}$. The same volume of trypsin digestion buffer was added again for an overnight incubation at $37^{\circ} \mathrm{C}$. Peptides were eluted from the S-Trap column first with $50 \mathrm{mM}$ TEAB spun through at $1,000 \times \mathrm{g}$, then with $50 \mathrm{mM}$ TEAB and $0.5 \%$ formic acid at $1,000 \times \mathrm{g}$, and finally with $50 \%$ acetonitrile in $0.5 \%$ formic acid at $4,000 \times \mathrm{g}$. These pooled elutions were dried in a vacuum concentrator and then re-suspended in $0.2 \%$ formic acid. The re-suspended peptide samples were desalted with stage tips generated in-house using $\mathrm{C} 18$ disks. They were subsequently dried again in a vacuum concentrator, and re-suspended in aqueous $0.2 \%$ formic acid containing "Hyper Reaction Monitoring" indexed retention time peptide standards (iRT, Biognosys).

\section{Mass Spectrometry Analysis}

Briefly, samples were analyzed by reverse-phase HPLC-ESI-MS/MS using an Eksigent Ultra Plus nano-LC 2D HPLC system (Dublin, CA) with a cHiPLC system (Eksigent) which was directly connected to a quadrupole time-of-flight (QqTOF) TripleTOF 6600 mass spectrometer (SCIEX, Concord, CAN). After injection, peptide mixtures were loaded onto a C18 pre-column chip (200 $\mu \mathrm{m} \times 0.4 \mathrm{~mm}$ ChromXP C18-CL chip, $3 \mu \mathrm{m}, 120 \AA$, SCIEX) and washed at $2 \mu \mathrm{l} / \mathrm{min}$ for $10 \mathrm{~min}$ with the loading solvent $\left(\mathrm{H}_{2} \mathrm{O} / 0.1 \%\right.$ formic acid) for desalting. Subsequently, peptides were transferred to the $75 \mu \mathrm{m} \times 15 \mathrm{~cm}$ ChromXP C18-CL chip, $3 \mu \mathrm{m}$, $120 \AA$, (SCIEX), and eluted at a flow rate of $300 \mathrm{~nL} / \mathrm{min}$ with a $3 \mathrm{~h}$ gradient using aqueous and acetonitrile solvent buffers.

Data-dependent acquisitions: For peptide and protein identifications the mass spectrometer was operated in data-dependent acquisition (DDA) mode, where the 30 most abundant 
precursor ions from the survey MS1 scan (250 msec) were isolated at $1 \mathrm{~m} / \mathrm{z}$ resolution for collision induced dissociation tandem mass spectrometry (CID-MS/MS, $100 \mathrm{msec}$ per MS/MS, 'high sensitivity' product ion scan mode) using the Analyst 1.7 (build 96) software with a total cycle time of $3.3 \mathrm{sec}$ as previously described (67)

Data Processing: Mass spectrometric data-dependent acquisitions (DDA) were analyzed using the database search engine ProteinPilot (SCIEX 5.0 revision 4769) allowing for biological modifications and with 'emphasis' on succinylation. A fasta file was generated appending the viral NSP14 protein sequence to the human proteome fasta file. A confidence score threshold of 99 was set to filter for high quality peptide identifications. Identified protein and peptide results are provided in Supplementary Table S1.

\section{SARS-CoV-2 virus culture and Infections}

SARS-CoV-2 isolate USA-WA1/2020 (BEI NR-52281) was used for all infection studies. All live virus experiments were performed in a Biosafety Level 3 laboratory. SARS-CoV-2 stocks were propagated in Vero-E6 cells, and their sequences were verified by next-generation sequencing. Viral stock titer was measured by plaque assays.

For infection experiments, A549-ACE2 or Calu3 cells were seeded into 12- or 24-well plates and rested for at least 24 hours prior to infection. At the time of infection, medium containing compound and/or viral inoculum ( $\mathrm{MOI} 0.01$ or 0.1 ) was added to the cells. After 3 days, the supernatant was collected and mixed with two volumes of RNA STAT-60 extraction buffer (AMSbio, UK). Cells were similarly harvested by adding RNA STAT-60 extraction buffer. Samples were stored at $-80^{\circ} \mathrm{C}$.

Infections of HCT-8 cells with HCoV-OC43 were performed similarly.

\section{Plaque assays}

Viral inoculations were harvested from experiments and serially diluted in DMEM (Corning). At the time of infection, the media on Vero-E6 cells were replaced with viral inoculation for 1 hour. After the 1-hour absorption period, 2.5\% Avicel (Dupont, RC-591 was layered on the cells and incubated for 72 hours. Then, Avicel was removed and cells were fixed in $10 \%$ formalin for 1 hour, stained with crystal violet for 10 minutes, and washed multiple times with water. Plaques were counted and averaged from two technical replicates.

\section{RNA extraction and RT-qPCR}

RNA in cells and supernatants resuspended in RNA STAT-60 buffer was extracted using Direct-zol RNA Miniprep kit (ZymoResearch, USA). For extraction from the supernatant, RNA was eluted in $20 \mu \mathrm{L}$ of water, and $18 \mu \mathrm{L}$ was directly used for reverse-transcription. RNA extracted from cells was DNAse-treated, eluted in $30 \mu \mathrm{L}$ quantified by Nanodrop, and $2 \mu \mathrm{g}$ of RNA was used reverse-transcription. Reverse-transcription was performed using the High-Capacity cDNA Reverse Transcription Kit (ThermoFisher), and quantitative PCR was done using a BioRad qPCR machine and Sybr Green (ThermoFisher). RT-qPCRs were normalized using the $\Delta \Delta \mathrm{Ct}$ method with the reference genes Actin and Gapdh. qPCR primers 
are given in Supplementary Table S3. Missing data points in RT-qPCR figures represent samples where RNA extraction was of poor quality. No outliers were removed.

\section{Statistics and reproducibility}

Experiments were carried out in multiple replicates. For affinity-purification and western blot data, one representative experiment out of several replicates is shown. For statistical analysis of RT-qPCR data, we used ordinary one-way ANOVA, followed by Holm-Šidák multiple comparisons test, with a single pooled variance. Plaque assay data do not satisfy the normality condition required for parametric tests, but are closer to a lognormal distribution (68). As a consequence, statistical tests on plaque assay data were performed on log-transformed data. To correct for batch effects between experiments performed on different days, plaque assay data were standardized. A pooled variance from all plaque assays was calculated, and for each data point, a Z-score was calculated using this pooled variance. The average of WT or DMSO-treated samples was used as a reference. This Z-score corrected for batch effect, and we used one-way ANOVA followed by Holm-Sidák's multiple-comparison test to analyze the effect of Sirtuin knockout or inhibition. Analyses were run using GraphPad Prism version 9.1.2 for macOS (GraphPad Software, USA, www.graphpad.com). Exact p-values and summaries are reported in the text and figures, respectively.

\section{RNA-sequencing preparation and analysis}

Library preparation and sequencing were performed by the DNA Technologies and Expression Analysis Core at the UC Davis Genome Center (Davis, CA, USA), supported by NIH Shared Instrumentation Grant 1S100D010786-01. Strand-specific and barcode indexed RNA-seq libraries were generated from $500 \mathrm{ng}$ of total RNA each, after poly-A enrichment, using the Kapa mRNA-seq Hyper kit (Kapa Biosystems-Roche, Basel, Switzerland), following the instructions of the manufacturer. The fragment size distribution of the libraries was verified via micro-capillary gel electrophoresis on a LabChip GX system (PerkinElmer, Waltham, MA). The libraries were quantified by fluorometry on a Qubit instrument (LifeTechnologies, Carlsbad, CA) and pooled in equimolar ratios. The pool was quantified by qPCR with a Kapa Library Quant kit (Kapa Biosystems) and sequenced on an Illumina NovaSeq 6000 (Illumina, San Diego, CA) with paired-end 150-bp reads.

Paired-end sequencing reads were mapped to a composite human/SARS-CoV-2 genome using Subread Aligner v2.0.3 (69). A genome index was constructed using GRCh38 genome build with Gencode v38 annotation of the transcriptome, and Genbank MT246667.1 for the sequence of SARS-CoV-2, USA/WA-1/2020 isolate. Reads mapping to annotated genes were counted using Subread featurecount v2.0.3 (70). Downstream analyses were performed with R. Differential gene expression analysis was done with DEseq2, which was also used to generate normalized gene counts (71). Low-expressed genes with less than three counts in at least three out of 16 samples were excluded from downstream analysis. q-Values were calculated using the q-value R package v2.24.0. For DEseq2 analysis, we used a one-factor design with four groups (WT mock, KO mock, WT infected and KO infected) and then 
likelihood ratio testing (LRT) to find all genes that were differentially expressed between at least two groups ( $q$-value threshold $<0.01$ ), and with a basemean expression $>15$. Consensus clustering of the 3221 differentially expressed genes was performed with the degPatterns function of R DEGreport package v1.28.0, using default parameters and rlog-transformed counts. This generated eight clusters. Over-representation of biological gene sets in the gene clusters was investigated using the R clusterProfiler package and enricher function (72). Gene sets were downloaded from the MSIG data bank via the msigdbr R-project package, including "Hallmark," and "Reactome". (73). Gene sets were considered significantly enriched in a cluster if $q$ values were $<0.05$. For analysis of restriction factors in figure 6 , we first selected genes in clusters 1 to 8 that belonged to the hallmark curated data set "Interferon Alpha response". We complemented this list with additional genes from clusters 7 and 8 that belonged to the "Interferon Gamma response", "Inflammatory response" and "TNFa signaling via NfKB" hallmark datasets, and finally added genes that we manually identified as potential restriction factors from literature searches. Average log2 fold-change compared to mock-infected WT was calculated and plotted, as well as the q-value between mock-infected WT and KO. Code developed for this study is available at https://github.com/mariuswalter/SIRT5_paper. This analysis relied heavily on code made available by the laboratory of Denis Goldfarb (https://github.com/GoldfarbLab/H522_paper_figures), and described in ref (45).

\section{Data and code availability}

The data supporting the findings of this study are available within the paper and its Supplementary files. RNA-seq data have been deposited to the GEO repository (GSE188382). Code developed for RNA-seq analysis is available on Github

(github.com/mariuswalter/SIRT5_paper). Mass spectrometric raw data have been deposited to the MassIVE repository (MSV000088589) and are also available at ProteomeXchange (PXD030530). Plasmids, viruses and other reagents developed in this study are available upon request and subject to standard material transfer agreements with the Buck Institute. Any other relevant data are available upon reasonable request.

\section{Acknowledgments}

We thank the QCRG Virology group at UCSF for technical and conceptual help, as well as members of the Verdin and Ott lab, in particular Rebeccah Riley, Rosalba Perrone and Anthony Covarrubias. We thank Michelle Moritz at UCSF for attempting co-purification experiments in E. coli. We thank David Gordon for quickly sharing the plasmid library early in the study and Max Greenberg at Paris University for sharing the Transposase plasmid.

This study was funded through institutional support from the Buck Institute for Research on Aging. IPC received support from the NIH (F31 Al164671-01). M.O. gratefully acknowledges support through gifts by Pamela and Edward Taft, and the Roddenberry Foundation. We acknowledge the support of instrumentation from the NCRR shared instrumentation grant 
1S10 OD016281 (Buck Institute) for Mass Spectrometry Analysis, and the NIH Shared Instrumentation Grant 1S100D010786-01 (UC Davis) for RNA-sequencing.

\section{Author contributions}

MW and EV designed the study and analyzed the data. MW, IJK, OB, AC performed molecular biology experiments. IPC, AVG, and JMH performed SARS-CoV-2 assays and analyses. VL and JDG provided purified Nsp14 proteins. SS and BS performed mass spectrometry experiments and analysis. MW analyzed RNA-seq data. EV and OT supervised and funded the project. M.W. and E.V. wrote the manuscript with input from all authors.

\section{Competing interests}

Authors declare no competing interests. 


\section{References}

1. D. E. Gordon, et al., A SARS-CoV-2 protein interaction map reveals targets for drug repurposing. Nature 583, 459-468 (2020).

2. D. E. Gordon, et al., Comparative host-coronavirus protein interaction networks reveal pan-viral disease mechanisms. Science $\mathbf{3 7 0}$ (2020).

3. J. Li, et al., Virus-Host Interactome and Proteomic Survey Reveal Potential Virulence Factors Influencing SARS-CoV-2 Pathogenesis. Med (N Y) 2, 99-112.e7 (2021).

4. E. M. N. Laurent, Y. Sofianatos, A. Komarova, J. P. Gimeno, Global BiolD-based SARS-CoV-2 proteins proximal interactome unveils novel ties between viral polypeptides and host factors involved in multiple COVID19 .... BioRxiv (2020).

5. E. Minskaia, et al., Discovery of an RNA virus $3^{\prime}->5^{\prime}$ exoribonuclease that is critically involved in coronavirus RNA synthesis. Proc. Natl. Acad. Sci. U. S. A. 103, 5108-5113 (2006).

6. Y. Chen, et al., Functional screen reveals SARS coronavirus nonstructural protein nsp14 as a novel cap N7 methyltransferase. Proc. Natl. Acad. Sci. U. S. A. 106, 3484-3489 (2009).

7. Y. Ma, et al., Structural basis and functional analysis of the SARS coronavirus nsp14-nsp10 complex. Proc. Natl. Acad. Sci. U. S. A. 112, 9436-9441 (2015).

8. L. Yan, et al., Coupling of N7-methyltransferase and 3'-5' exoribonuclease with SARS-CoV-2 polymerase reveals mechanisms for capping and proofreading. Cell 184, 3474-3485.e11 (2021).

9. F. Ferron, et al., Structural and molecular basis of mismatch correction and ribavirin excision from coronavirus RNA. Proc. Natl. Acad. Sci. U. S. A. 115, E162-E171 (2018).

10. M. Bouvet, et al., RNA 3 '-end mismatch excision by the severe acute respiratory syndrome coronavirus nonstructural protein nsp10/nsp14 exoribonuclease complex. Proc. Natl. Acad. Sci. U. S. A. 109, 9372-9377 (2012).

11. C. Liu, et al., Structural basis of mismatch recognition by a SARS-CoV-2 proofreading enzyme. Science (2021) https:/doi.org/10.1126/science.abi9310 (July 28, 2021).

12. L. D. Eckerle, X. Lu, S. M. Sperry, L. Choi, M. R. Denison, High fidelity of murine hepatitis virus replication is decreased in nsp14 exoribonuclease mutants. J. Virol. 81, 12135-12144 (2007).

13. L. D. Eckerle, et al., Infidelity of SARS-CoV Nsp14-exonuclease mutant virus replication is revealed by complete genome sequencing. PLoS Pathog. 6, e1000896 (2010).

14. A. E. Gorbalenya, L. Enjuanes, J. Ziebuhr, E. J. Snijder, Nidovirales: evolving the largest RNA virus genome. Virus Res. 117, 17-37 (2006).

15. C. Lauber, et al., The footprint of genome architecture in the largest genome expansion in RNA viruses. PLoS Pathog. 9, e1003500 (2013).

16. N. S. Ogando, et al., The Enzymatic Activity of the nsp14 Exoribonuclease Is Critical for Replication of MERS-CoV and SARS-CoV-2. J. Virol. 94 (2020).

17. J. C.-C. Hsu, M. Laurent-Rolle, J. B. Pawlak, C. B. Wilen, P. Cresswell, Translational shutdown and evasion of the innate immune response by SARS-CoV-2 NSP14 protein. Proc. Natl. Acad. Sci. U. S. A. 118 (2021).

18. M. Hayn, et al., Systematic functional analysis of SARS-CoV-2 proteins uncovers viral innate immune antagonists and remaining vulnerabilities. Cell Rep. 35, 109126 (2021).

19. T. Li, et al., SARS-CoV-2 Nsp14 activates NF-KB signaling and induces IL-8 upregulation. bioRxiv (2021) https:/doi.org/10.1101/2021.05.26.445787.

20. J. Gribble, et al., The coronavirus proofreading exoribonuclease mediates extensive viral recombination. PLoS Pathog. 17, e1009226 (2021).

21. R. H. Houtkooper, E. Pirinen, J. Auwerx, Sirtuins as regulators of metabolism and healthspan. Nat. Rev. Mol. Cell Biol. 13, 225-238 (2012).

22. S.-I. Imai, L. Guarente, It takes two to tango: NAD+ and sirtuins in aging/longevity control. NPJ Aging Mech Dis 2, 16017 (2016).

23. J. Du, et al., Sirt5 is a NAD-dependent protein lysine demalonylase and desuccinylase. Science 334, 806-809 (2011).

24. M. Tan, et al., Lysine glutarylation is a protein posttranslational modification regulated by SIRT5. 
Cell Metab. 19, 605-617 (2014).

25. M. J. Rardin, et al., SIRT5 regulates the mitochondrial lysine succinylome and metabolic networks. Cell Metab. 18, 920-933 (2013).

26. Y. Nishida, et al., SIRT5 Regulates both Cytosolic and Mitochondrial Protein Malonylation with Glycolysis as a Major Target. Mol. Cell 59, 321-332 (2015).

27. S. Kumar, D. B. Lombard, Functions of the sirtuin deacylase SIRT5 in normal physiology and pathobiology. Crit. Rev. Biochem. Mol. Biol. 53, 311-334 (2018).

28. J. Yu, et al., Metabolic characterization of a Sirt5 deficient mouse model. Sci. Rep. 3, 2806 (2013).

29. N. C. Yeo, et al., An enhanced CRISPR repressor for targeted mammalian gene regulation. Nat. Methods 15, 611-616 (2018).

30. X. Liu, et al., SIRT5 impairs aggregation and activation of the signaling adaptor MAVS through catalyzing lysine desuccinylation. EMBO J. 39, e103285 (2020).

31. X. Yang, et al., SHMT2 Desuccinylation by SIRT5 Drives Cancer Cell Proliferation. Cancer Res. 78, 372-386 (2018).

32. Y. Xiangyun, et al., Desuccinylation of pyruvate kinase M2 by SIRT5 contributes to antioxidant response and tumor growth. Oncotarget 8, 6984-6993 (2017).

33. A. M. Davenport, F. M. Huber, A. Hoelz, Structural and functional analysis of human SIRT1. J. Mol. Biol. 426, 526-541 (2014).

34. N. Rajabi, et al., Mechanism-Based Inhibitors of the Human Sirtuin 5 Deacylase: Structure-Activity Relationship, Biostructural, and Kinetic Insight. Angew. Chem. Int. Ed Engl. 56, 14836-14841 (2017).

35. E. Verdin, $N A D^{+}$in aging, metabolism, and neurodegeneration. Science 350, 1208-1213 (2015).

36. A. J. Covarrubias, et al., Senescent cells promote tissue NAD+ decline during ageing via the activation of CD38+ macrophages. Nat Metab 2, 1265-1283 (2020).

37. M. Bouhaddou, et al., The Global Phosphorylation Landscape of SARS-CoV-2 Infection. Cell 182, 685-712.e19 (2020).

38. L. Polletta, et al., SIRT5 regulation of ammonia-induced autophagy and mitophagy. Autophagy 11, 253-270 (2015).

39. Y.-Q. Wang, et al., Sirtuin 5 contributes to colorectal carcinogenesis by enhancing glutaminolysis in a deglutarylation-dependent manner. Nat. Commun. 9, 545 (2018).

40. Y. L. N. Abril, et al., Pharmacological and genetic perturbation establish SIRT5 as a promising target in breast cancer. Oncogene 40, 1644-1658 (2021).

41. L. Chang, et al., SIRT5 promotes cell proliferation and invasion in hepatocellular carcinoma by targeting E2F1. Mol. Med. Rep. 17, 342-349 (2018).

42. D. Blanco-Melo, et al., Imbalanced Host Response to SARS-CoV-2 Drives Development of COVID-19. Cell 181, 1036-1045.e9 (2020).

43. T. Yamada, et al., RIG-I triggers a signaling-abortive anti-SARS-CoV-2 defense in human lung cells. Nat. Immunol. 22, 820-828 (2021).

44. Y. Kasuga, B. Zhu, K.-J. Jang, J.-S. Yoo, Innate immune sensing of coronavirus and viral evasion strategies. Exp. Mol. Med. 53, 723-736 (2021).

45. M. Puray-Chavez, et al., Systematic analysis of SARS-CoV-2 infection of an ACE2-negative human airway cell. Cell Rep. 36, 109364 (2021).

46. C. Sanders, P. Thomas, MMP-7 promotes host recovery and lung function to influenza virus infection (VIR2P.1016). The Journal of Immunology 192, 75.5-75.5 (2014).

47. T. H. Flo, et al., Lipocalin 2 mediates an innate immune response to bacterial infection by sequestrating iron. Nature 432, 917-921 (2004).

48. C. Ehre, et al., Overexpressing mouse model demonstrates the protective role of Muc5ac in the lungs. Proc. Natl. Acad. Sci. U. S. A. 109, 16528-16533 (2012).

49. A. D. Arslan, et al., Human SLFN5 is a transcriptional co-repressor of STAT1-mediated interferon responses and promotes the malignant phenotype in glioblastoma. Oncogene 36, 6006-6019 (2017). 
50. J. Niemelä, et al., IFN-alpha induced adenosine production on the endothelium: a mechanism mediated by CD73 (ecto-5'-nucleotidase) up-regulation. J. Immunol. 172, 1646-1653 (2004).

51. F. Blanc, et al., Targeting host calpain proteases decreases influenza A virus infection. Am. J. Physiol. Lung Cell. Mol. Physiol. 310, L689-99 (2016).

52. A. R. Massey, J. D. Beckham, Alpha-Synuclein, a Novel Viral Restriction Factor Hiding in Plain Sight. DNA Cell Biol. 35, 643-645 (2016).

53. H.-S. Kwon, et al., Human immunodeficiency virus type 1 Tat protein inhibits the SIRT1 deacetylase and induces T cell hyperactivation. Cell Host Microbe 3, 158-167 (2008).

54. S. Pagans, et al., SIRT1 regulates HIV transcription via Tat deacetylation. PLoS Biol. 3, e41 (2005).

55. T. Heinonen, et al., Sirtuin 5 Deficiency Does Not Compromise Innate Immune Responses to Bacterial Infections. Front. Immunol. 9, 2675 (2018).

56. E. Koyuncu, et al., Sirtuins are evolutionarily conserved viral restriction factors. MBio 5 (2014).

57. F. Hou, et al., MAVS forms functional prion-like aggregates to activate and propagate antiviral innate immune response. Cell 146, 448-461 (2011).

58. Y.-Z. Fu, et al., SARS-CoV-2 membrane glycoprotein $\mathrm{M}$ antagonizes the MAVS-mediated innate antiviral response. Cell. Mol. Immunol. 18, 613-620 (2021).

59. C.-S. Shi, et al., SARS-coronavirus open reading frame-9b suppresses innate immunity by targeting mitochondria and the MAVS/TRAF3/TRAF6 signalosome. J. Immunol. 193, 3080-3089 (2014).

60. E. C. Freundt, L. Yu, E. Park, M. J. Lenardo, X.-N. Xu, Molecular determinants for subcellular localization of the severe acute respiratory syndrome coronavirus open reading frame $3 \mathrm{~b}$ protein. J. Virol. 83, 6631-6640 (2009).

61. Y. Chen, Z. Zhou, W. Min, Mitochondria, Oxidative Stress and Innate Immunity. Front. Physiol. 9, 1487 (2018).

62. T. Weichhart, M. Hengstschläger, M. Linke, Regulation of innate immune cell function by mTOR. Nat. Rev. Immunol. 15, 599-614 (2015).

63. J. Guan, et al., Sirtuin 5 regulates the proliferation, invasion and migration of prostate cancer cells through acetyl-CoA acetyltransferase 1. J. Cell. Mol. Med. 24, 14039-14049 (2020).

64. M. A. Horlbeck, et al., Compact and highly active next-generation libraries for CRISPR-mediated gene repression and activation. Elife 5, e19760 (2016).

65. A. Vallejo-Gracia, et al., FOXO1 promotes HIV latency by suppressing ER stress in T cells. Nat Microbiol 5, 1144-1157 (2020).

66. T. Tezil, et al., Lifespan-increasing drug nordihydroguaiaretic acid inhibits p300 and activates autophagy. NPJ Aging Mech Dis 5, 7 (2019).

67. D. G. Christensen, et al., Identification of Novel Protein Lysine Acetyltransferases in Escherichia coli. MBio 9 (2018).

68. M. Walter, E. Verdin, Viral gene drive in herpesviruses. Nat. Commun. 11, 4884 (2020).

69. Y. Liao, G. K. Smyth, W. Shi, The Subread aligner: fast, accurate and scalable read mapping by seed-and-vote. Nucleic Acids Res. 41, e108 (2013).

70. Y. Liao, G. K. Smyth, W. Shi, featureCounts: an efficient general purpose program for assigning sequence reads to genomic features. Bioinformatics 30, 923-930 (2014).

71. M. I. Love, W. Huber, S. Anders, Moderated estimation of fold change and dispersion for RNA-seq data with DESeq2. Genome Biol. 15, 550 (2014).

72. G. Yu, L. G. Wang, Y. Han, Q. Y. He, clusterProfiler: an R package for comparing biological themes among gene clusters. OMICS (2012).

73. A. Liberzon, et al., Molecular signatures database (MSigDB) 3.0. Bioinformatics 27, 1739-1740 (2011). 


\section{Figure Legends}

\section{Figure 1: SARS-CoV-2 Nsp14 interacts with human SIRT5}

A. Cartoon representation of the protein structure of Nsp14/Nsp10 (PDB 7N0B) and SIRT5 (PDB 3YIR) shows the Nsp14 N-terminal ExoN domain and C-terminal MTase domain. B. Affinity-purification of Nsp14-strep and co-purification of endogenous SIRT5 after transfection in HEK293T cells, as shown by western blot. C. Immunofluorescence of transfected Nsp14-Strep and endogenous SIRT5 in A549 cells. D. CETSA in HEK293T cells transfected with Nsp14-step and/or SIRT5, showing an increase in the stability of SIRT5 and Nsp14 by western blot. E. Western blot showing the absence of SIRT5 in Sirt5-KD HEK293T cells. F. Strep-tag affinity-purification or Flag-tag immunoprecipitation, followed by western blot, after transfection with Nsp14-strep, Nsp10-flag and SIRT5 expression constructs. SIRT5 does not interact with Nsp10. $0.5 \mu \mathrm{g}$ of each construct or of empty control plasmids were transfected in Sirt5-KD HEK293T cells in a six-well plate. G. Strep-tag affinity-purification and western blot after transfection of Nsp14-strep, SIRT5 and increasing concentrations of Nsp10-tag indicates competitive binding of SIRT5 and Nsp10. $0.5 \mu \mathrm{g}$ of Nsp14-strep and SIRT5 plasmid were used in a 6-well plate, with $0,0.5,1$ or $2 \mu \mathrm{g}$ of Nsp10-Flag.

Figure 2: SIRT5 catalytic activity is necessary to interact with Nsp14.

A. Cartoon representation of the protein structure of SIRT5 catalytic site in complex with cofactor NAD and succinylated lysine substrate (SuK), showing conserved residues mutated in panel B. B. Strep-tag affinity-purification and western blot after transfection of Sirt5-KD HEK293T cells with Nsp14-strep and SIRT5 catalytic mutants, showing that the interaction with Nsp14 is lost in several mutants. C. Strep-tag affinity-purification and western blot after transfection with Nsp14-strep and SIRT5, in Sirt5-KD HEK293T cells incubated with increasing concentrations of SIRT5 inhibitor Sirt5-i. High concentrations of Sirt5-i prevent the interaction. D. Strep-tag affinity-purification and western blot after transfection with Nsp14-strep and SIRT5, in Sirt5-KD HEK293T cells incubated with NAMPT FK866 inhibitor (low cellular NAD), FK866 and NMN, or NMN alone (high cellular NAD). SIRT 5 binding strength correlated with NAD levels. E. Pan-acetylation, malonylation and succinylation in Sirt5-KD HEK293T total or Strep-purified proteins, after transfection with Nsp14-Strep, GFP-strep control and/or SIRT5. No specific lysine modifications could be detected. F. Summary of mass spectrometry experiments. Nsp14-strep proteins purified from Sirt5-KD HEK293T, with or without co-transfection with SIRT5, were analyzed by mass spectrometry. No acetylation, malonylation or succinylation modifications could be detected.

\section{Figure 3: SARS-CoV-2 Nsp14 interacts with human SIRT1.}

A. Co-purification of endogenous sirtuins SIRT1, 2 , 3, 6 and 7 after transfection of Nsp14-strep in HEK293T cells, as shown by western blot. Loading and purification controls are the same as in Fig. 1B. B. Strep-tag affinity-purification and western blot after transfection of HEK293T cells with Nsp14-strep and SIRT1 WT and H355Y catalytic mutant, showing that the interaction with Nsp14 is lost in H355Y mutant. C. Strep-tag affinity-purification and western blot after transfection with Nsp14-strep and SIRT1, in HEK293T cells incubated with increasing concentrations of SIRT1 inhibitor Ex-527. High concentrations of Ex-527 prevent the interaction. D-E. Strep-tag affinity-purification and 
western blot after transfection with Nsp14-strep and SIRT1, in HEK293T cells incubated with increasing concentrations of SIRT1 specific activator SRT1720 (D) or non-specific activator resveratrol (E). Both drugs were cytotoxic at high concentration and didn't affect SIRT5 binding. F. In vitro desuccinylation activity of purified SIRT5 incubated with increasing concentrations of Nsp14, showing no effect. G. In vitro methyltransferase activity of purified Nsp14 incubated with increasing concentrations of SIRT5, showing no specific effect. Unmethylated GpppG cap-analog was used as a substrate.

\section{Figure 4: SIRT5 is a proviral factor}

A. Western blot showing the absence of SIRT5 and SIRT1 in Sirt5- and Sirt1-KO A549-ACE2 cells, after CRISPR knockout. B. Decrease in cell-associated viral mRNA levels in Sirt5- and Sirt1-KO cells infected with SARS-Cov- 2 for 3 days at $\mathrm{MOI}=0.1$ or $\mathrm{MOI}=1$, as shown by RT-qPCR. Data show fold-changes compared to WT levels (at MOI=0.1). $n=4$. C. Decrease in viral titers in Sirt5- and Sirt1-KO cells infected with SARS-Cov-2 for 3 days at $\mathrm{MOI}=1$, as shown by plaque assay. $n=6$. D. Absence of cytotoxicity in A549 cells treated with Sirt5-i and Ex-527 inhibitor, as measured by flow cytometry. $n=4$. E. Decrease in cell-associated viral mRNA levels in A549-ACE2 cells infected with SARS-Cov-2 for 3 days at $\mathrm{MOI}=0.1$, and treated with SIRT 5 and SIRT1 inhibitors Sirt5-i and Ex-527, as shown by RT-qPCR. Data show fold-change compared to DMSO-treated levels. $n=6$. F. Decrease in viral titers in A549-ACE2 cells infected with SARS-Cov- 2 for 3 days at MOI $=0.1$, and treated with SIRT 5 and SIRT1 inhibitors Sirt5-i and Ex-527, as shown by plaque assay. $n=9$. G/H. Same as E. (with $n=4$ ), and F. $(n=13)$, using Calu3 cells. I. Statistical analysis of plaque assay data to account for the batch effect between experiments performed on different days. A standardized Z-score was calculated and plotted, showing a significant reduction of viral titers in A549-ACE2 KO cells and Calu3. J. Strep-tag affinity-purification and western blot after transfection of Sirt5-KD HEK293T cells with SIRT5 and Nsp14-strep from different coronaviruses, showing that the interaction with SIRT5 is specific to SARS-like coronaviruses. $\mathbf{K}$. Decrease in supernatant-associated viral mRNA levels in HCT-8 cells infected with HCoV-OC43 for 5 days at $\mathrm{MOI}=0.1$, and treated with SIRT5 inhibitor Sirt5-i, as shown by RT-qPCR. Data show fold-change compared to DMSO-treated levels. $n=4$. L. Decrease in viral titers in HCT-8 cells infected with $\mathrm{HCOV}-\mathrm{OC} 43$ for 5 days at $\mathrm{MOI}=0.1$, and treated with SIRT5 inhibitors Sirt5-i and Ex-527, as shown by plaque assay. $n=4$.

Data show mean and standard error of the mean (SEM) between biological replicates. RT-qPCR results were internally normalized with Gapdh and Actin reference genes. Viral titers after plaque assay are expressed in log-transformed PFU (plaque-forming unit) per $\mathrm{mL}$ of supernatant. Asterisks summarize the results of statistical tests. $*$ : $p<0.05, * *$ : $p<0.01, * \star *$ : $p<0.001, * \star \star *: p<0.0001$. For RT-qPCR data and plaque assay Z-scores, we used one-way ANOVAs followed by Holm-Šidák multiple comparisons test. For plaque assays, we used one-way ANOVAs followed by Holm-Šidák multiple comparisons test on log-transformed data.

\section{Figure 5: Sirt5-KO cells mount a stronger innate immune response}

RNA-seq analysis of WT and Sirt5-KO A549-ACE2 cells infected or mock-infected for 3 days with SARS-CoV-2 at $\mathrm{MOI}=1 . \mathrm{n}=4$. A. Volcano plots showing differentially expressed genes between the different conditions. Highlighted genes display a q-value $\mathrm{q}<0.05$ (green), log2 fold-change $>1$ (orange), or both (purple). Left panel: Sirt5-KO vs WT in mock-infected cells. 
Middle: Infected vs mock-infected WT cells. Right: Infected vs mock-infected Sirt5-KO cells.

B. Normalized gene count of SARS-CoV-2. C-D. Unsupervised clustering of the 3221 genes differentially expressed between at least two of the four conditions $(q<0.01)$. C: heatmap of normalized expression. D. Z-scores of differentially expressed genes as grouped by clustering. Colored lines represent quantification of an individual gene whereas solid black lines show the cluster Tukey boxplot. E. Enrichment analysis of biological gene sets in the identified gene clusters ( $C$ and $D)$.

Figure 6: Sirt5 knockout cells express a higher basal level of viral restriction factors

A. Expression heatmap of interferon-stimulated genes and other restriction factors, showing that mock-infected Sirt5-KO cells express higher basal levels of restriction factors, and that antiviral responses are stronger in Sirt5-KO cells. Data show mean log2 fold-change, compared to mock-infected WT, and the q-value between mock-infected WT and Sirt5-KO cells. Only genes differentially expressed between at least two conditions were included in the analysis $(q<0.01)$. B. Hypotheses for the role of the SIRT5/Nsp14 interaction during SARS-CoV-2 infection. In model 1, Nsp14 could enhance SIRT5 activity, which would decrease innate immune responses and favor viral replication. In model 2, Nsp14 could redirect SIRT 5 to novel targets, potentially in the replication-transcription complex, where SIRT5 could deacylate other viral proteins. In model 3, the Nsp14/SIRT5 complex could be primarily involved in mRNA cap methylation. Absence or inhibition of SIRT5 would lead to incomplete cap methylation and stronger immune recognition of viral mRNA.

\section{Supplementary Figure 1: Characterization of inhibitors.}

A. SIRT5 in vitro desuccinylation activity in the presence of Sirt5-i inhibitor. $n=3$. B. Diagram of the NAD salvage pathway. Inhibition of NAMPT by FK866 inhibitor depletes cellular NMN and NAD levels. Supplementation by exogenous NMN rescues NAD.

\section{Supplementary Figure 2: Levels of viral restriction factors.}

A. Principal component analysis of RNA-seq samples shows that replicates are well separated based on knockout and infection status. B. Normalized gene count of interferon-stimulated genes and restriction factors, from Figure 6 


\section{Supplementary Table S3}

\section{CRISPR gRNAs (Knockout in A549-ACE2)}

\begin{tabular}{|l|c|c|c|}
\cline { 2 - 4 } \multicolumn{1}{c|}{} & gRNA 1 & gRNA 2 & gRNA 3 \\
\hline Sirt1 & CAAAGGAUAAUUCAGUGUCA & AUAGCCUUGUCAGAUAAGGA & UUGAUACAGGAAAUAUAUCC \\
\hline Sirt5 & GUGCAGCUCAUCGAUGUUCU & AGCCGAGUGUGAGACCCGGC & CCGGGACGGGUUGUGGGCAA \\
\hline
\end{tabular}

CRISPR genotyping primers (Knockout in A549-ACE2)

\begin{tabular}{|c|c|c|l|}
\cline { 2 - 4 } \multicolumn{1}{c|}{ Forward } & \multicolumn{1}{c|}{ Reverse } & \multicolumn{1}{c|}{ Sequencing } \\
\hline Sirt1 & GCATATGACAGCAACCGTCC & GCTTTATCTCCACTTCTCGATGG & $\begin{array}{l}\text { GTGTCGCATCCATCTAGATACT } \\
\text { TTAAAAT }\end{array}$ \\
\hline Sirt5 & GCATCTGCCATGTTGTTTGA & CTGAAACAGCAGGACAGGTG & $\begin{array}{l}\text { CATCTGCCATGTTGTTTGAACA } \\
\text { TAGT }\end{array}$ \\
\hline
\end{tabular}

\section{CRISPRi gRNAs (knockdown in HEK293T)}

\begin{tabular}{|l|l|}
\cline { 2 - 2 } \multicolumn{1}{c|}{} & \multicolumn{1}{c|}{ gRNA } \\
\hline Sirt5 & GGCGCTCCGGACCTGAGCCA \\
\hline Non-targeting control & GCTGCATGGGGCGCGAATCA \\
\hline
\end{tabular}

Sequences from Horlbeck et al., 2016 (64)

\section{qPCR primers}

\begin{tabular}{|l|l|l|}
\cline { 2 - 3 } \multicolumn{1}{c|}{} & \multicolumn{1}{c|}{ Forward } & \multicolumn{1}{c|}{ Reverse } \\
\hline SARS-CoV-2 gene N & CACATTGGCACCCGCAATC & GAGGAACGAGAAGAGGCTTG \\
\hline OC43 gene N & CCGACTAGGTTTCCGCCTGG & TCTGCTGGATGTGCGCGAAG \\
\hline Gapdh & TTCTACAATGAGCTGCGTGTG & GGGGTGTTGAAGGTCTCAAA \\
\hline Actin & CTGTTGCTGTAGCCAAATTCGT & ACCCACTCCTCCACCTTTGAC \\
\hline
\end{tabular}

Primary antibodies

\begin{tabular}{|l|l|}
\hline Strep-tag mouse & Qiagen \#34850 \\
\hline Flag-tag rabbit & Cell Signaling \#14793S \\
\hline Beta-tubulin rabbit & Cell Signaling \#2128S \\
\hline SIRT1 rabbit & Cell Signaling \#9475 \\
\hline SIRT2 rabbit & Cell Signaling \#12650 \\
\hline SIRT3 rabbit & Cell Signaling \#5490 \\
\hline SIRT5 rabbit & Cell Signaling 8779S \\
\hline SIRT6 rabbit & Cell Signaling \#12486 \\
\hline SIRT7 rabbit & Cell Signaling \#5360 \\
\hline Acetyllysine rabbit & PTM Biolab \# PTM-105 \\
\hline Succinyllysine rabbit & PTM Biolab \# PTM-401 \\
\hline
\end{tabular}




\begin{tabular}{|l|l|}
\hline Malonyllysine rabbit & PTM Biolab \# PTM-901 \\
\hline Anti-mouse IgG, HRP-linked & Cell Signaling \#7076 \\
\hline Anti-rabbit IgG, HRP-linked & Cell Signaling \#7074 \\
\hline
\end{tabular}

\section{Small-molecule inhibitors and activators}

\begin{tabular}{|l|l|}
\hline Sirt5 inhibitor 1 & Medchemexpress HY-112634 \\
\hline Sirt1 inhibitor Ex-527 & Sigma-Aldrich E7034-5MG \\
\hline NAMPT inhibitor FK866 & Sigma-Aldrich F8557 \\
\hline Resveratrol & Sigma-Aldrich R5010-100MG \\
\hline SRT1720 & Selleckchem S1129 \\
\hline
\end{tabular}


Walter et al., 2022 - SIRT5/Nsp14

A

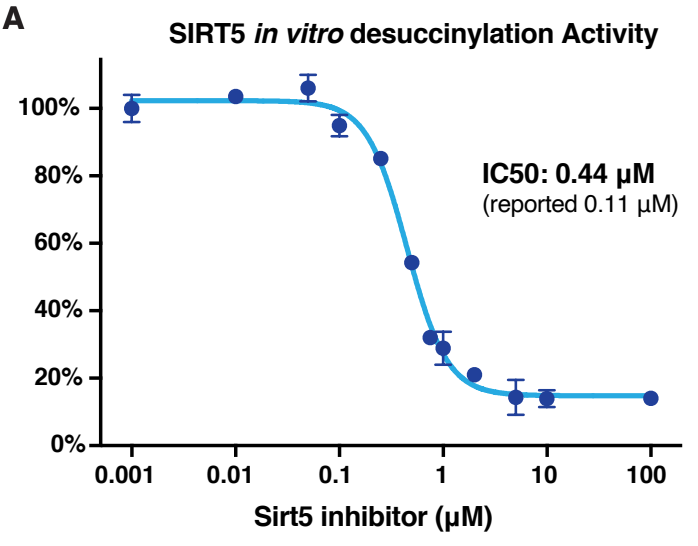

B

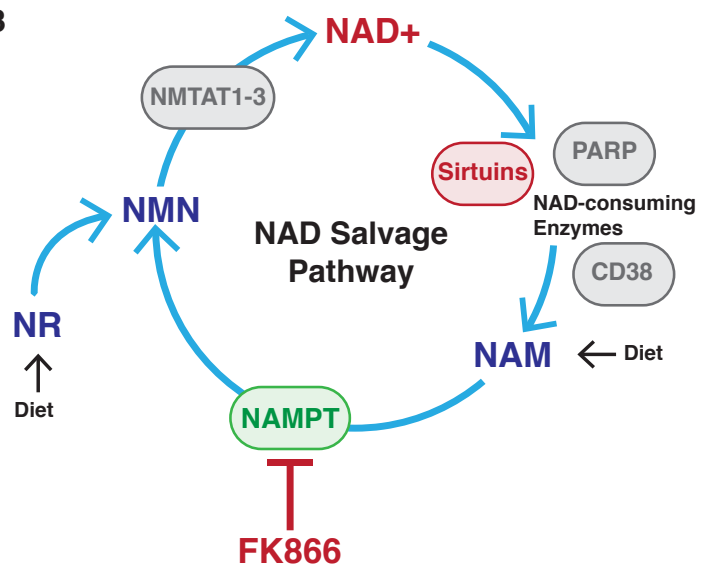

Supplementary Figure 1: Characterization of inhibitors.

A. SIRT5 in vitro desuccinylation activity in the presence of Sirt5-i inhibitor. $n=3$. B. Diagram of the NAD salvage pathway. Inhibition of NAMPT by FK866 inhibitor depletes cellular NMN and NAD levels. Supplementation by exogenous NMN rescues NAD.

Figure 2: Sirt5 interact through its catalytic domain

A. Left: Localizations of gene drive and GFP cassettes on hCMV genomes. UL/US indicates Unique Long/Short 
Walter et al., 2022 - SIRT5/Nsp14

A

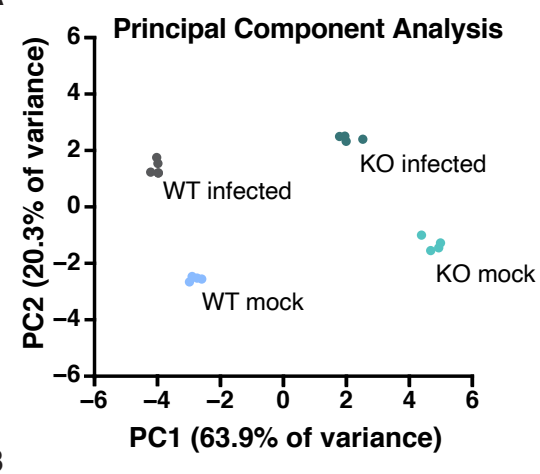

B

IFITM1

IFITM2
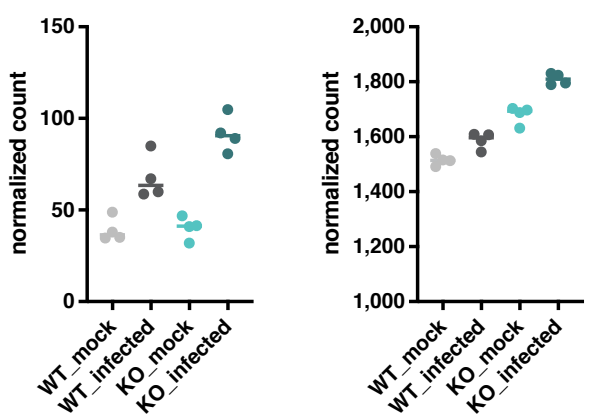

IFITM3

CXCL5

IL33

C1R

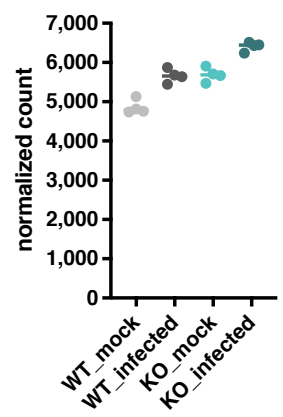

C1s
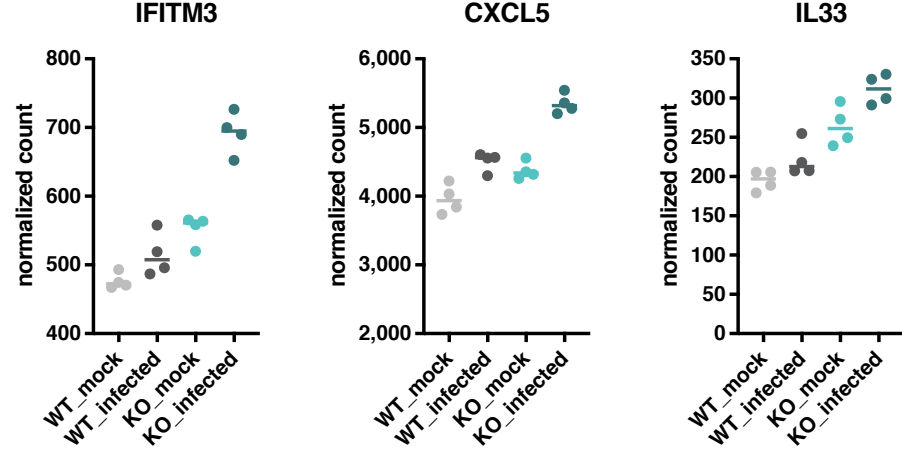

C3
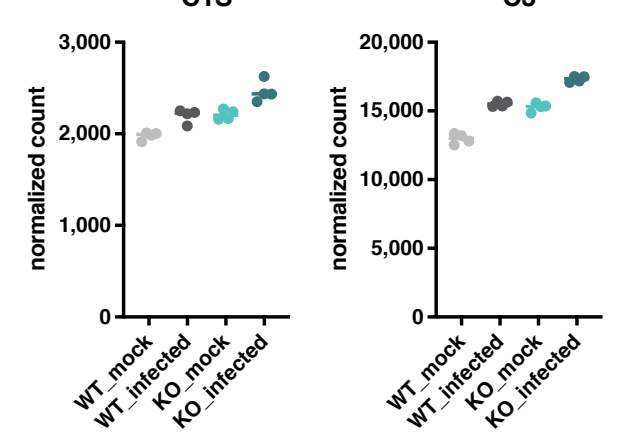

B2M

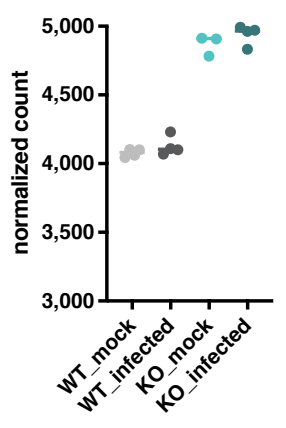

STAT3

SNCA

MUC5AC

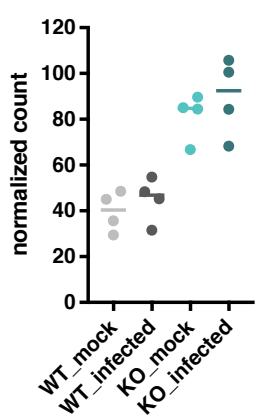

SLFN5

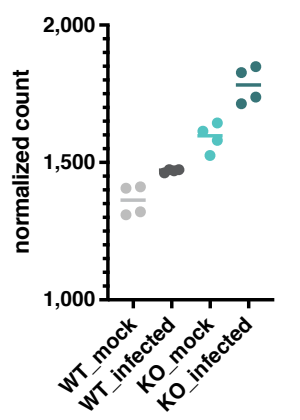

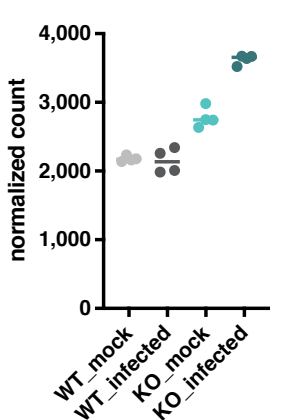

IRAK1

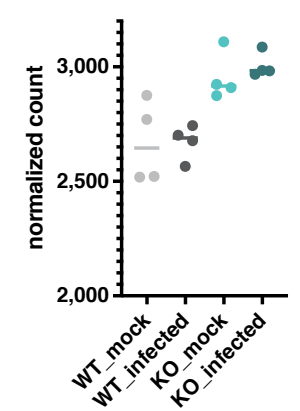

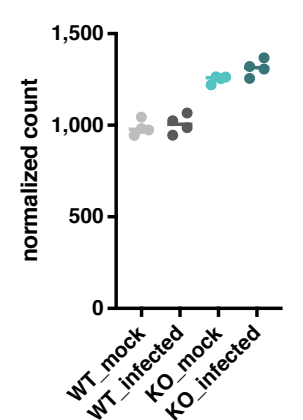

IFIT3

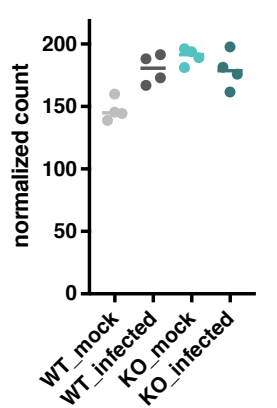

LCN2

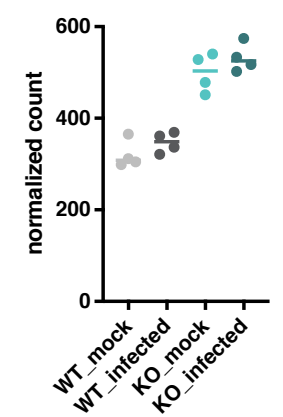

CD38

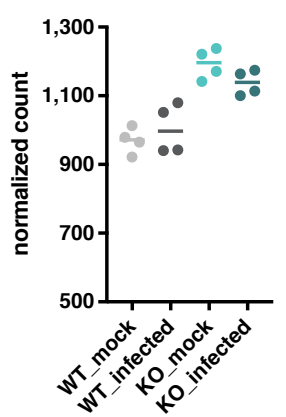

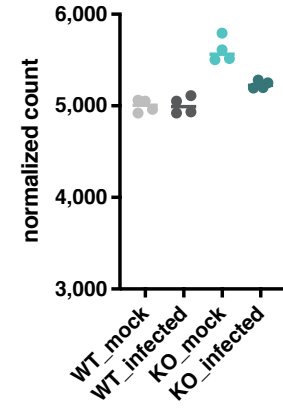

NT5E

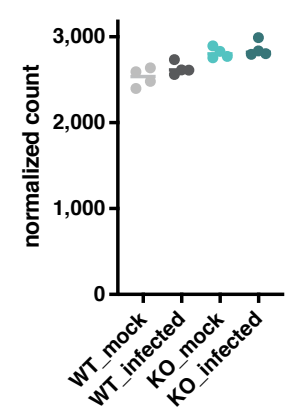

ALDH3A1

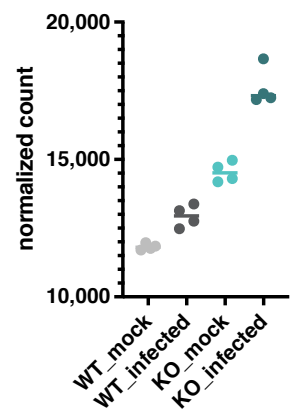

Supplementary Figure 2: Levels of viral restriction factors.

A. Principal component analysis of RNA-seq samples, showing that replicates are well separated based on knockout and infection status. B. Normalized gene count of interferon-stimulated genes and restriction factors, from Figure 6. 